\title{
General approach for solving the density gradient theory in the interfacial tension calculations
}

\section{Liang, Xiaodong; Michelsen, Michael Locht}

\section{Published in:}

Fluid Phase Equilibria

Link to article, DOI:

10.1016/j.fluid.2017.07.021

Publication date:

2017

Document Version

Peer reviewed version

Link back to DTU Orbit

\section{Citation (APA):}

Liang, X., \& Michelsen, M. L. (2017). General approach for solving the density gradient theory in the interfacial tension calculations. Fluid Phase Equilibria, 451, 79-90. https://doi.org/10.1016/j.fluid.2017.07.021

\section{General rights}

Copyright and moral rights for the publications made accessible in the public portal are retained by the authors and/or other copyright owners and it is a condition of accessing publications that users recognise and abide by the legal requirements associated with these rights.

- Users may download and print one copy of any publication from the public portal for the purpose of private study or research.

- You may not further distribute the material or use it for any profit-making activity or commercial gain

- You may freely distribute the URL identifying the publication in the public portal 


\section{Accepted Manuscript}

General approach for solving the density gradient theory in the interfacial tension calculations

Xiaodong Liang, Michael Locht Michelsen

PII:

S0378-3812(17)30289-3

DOI:

10.1016/j.fluid.2017.07.021

Reference: $\quad$ FLUID 11543

To appear in: Fluid Phase Equilibria

Received Date: 14 March 2017

Revised Date: 10 July 2017

Accepted Date: 28 July 2017

Please cite this article as: X. Liang, M.L. Michelsen, General approach for solving the density gradient theory in the interfacial tension calculations, Fluid Phase Equilibria (2017), doi: 10.1016/ j.fluid.2017.07.021.

This is a PDF file of an unedited manuscript that has been accepted for publication. As a service to our customers we are providing this early version of the manuscript. The manuscript will undergo copyediting, typesetting, and review of the resulting proof before it is published in its final form. Please note that during the production process errors may be discovered which could affect the content, and all legal disclaimers that apply to the journal pertain. 


\title{
General approach for solving the density gradient
}

\section{theory in the interfacial tension calculations}

\author{
Xiaodong Liang ${ }^{*}$, Michael Locht Michelsen \\ Center for Energy Resources Engineering (CERE), Department of Chemical and Biochemical \\ Engineering, Technical University of Denmark, 2800 Kgs. Lyngby, Denmark
}

Keywords: Density gradient theory, Interfacial tension, Direct optimization, CPA, PC-SAFT

Within the framework of the density gradient theory, the interfacial tension can be calculated by finding the density profiles that minimize an integral of two terms over the system of infinite width. It is found that the two integrands exhibit a constant difference along the interface for a finite planar interface, and this difference becomes smaller as the interface width increases. These findings naturally lead to a solution procedure that consists of an inner loop and an outer loop for calculating the interfacial tension of a planar interface. The outer loop deals with the relationship between the interfacial tension and the interface width, and it permits us to obtain accurate results from finite width calculations. The inner loop minimizes the interfacial tension for a given interface width by adjusting the density profiles, in which the integrals are evaluated by a combination of Gauss-Lobatto quadrature and Lagrange interpolation based polynomial approximation. A better approximation of the interfacial tension is derived by a path integration along the density profiles. These strategies enable us to obtain accurate solutions with looser tolerance criteria and a fewer number of thermodynamic property evaluations compared to other methods. The performance of the algorithm with recommended parameters is analyzed for various systems, and the efficiency is further compared with the geometric-mean density gradient theory, which only needs to solve nonlinear algebraic equations. The results show that the algorithm is only 5-10 times less efficient than solving the geometric-mean density gradient theory. 


\section{Introduction}

The density gradient theory (DGT) is a popular framework in the interfacial tension calculations [1-22]. The DGT was proposed by van der Waals [23] and reformulated by Cahn and Hilliard [24]. The DGT considers that the Helmholtz free energy density of an inhomogeneous fluid consists of two terms: the first term represents the Helmholtz free energy density of a homogeneous fluid modelled by the same thermodynamic model which is used for describing the bulk phases. The second term is expressed as the product of the so-called influence parameter and the square of the density gradient. We refer the first term as an energy term and the second term as a gradient term in this work.

The total Helmholtz free energy of the inhomogeneous system is the integral of the Helmholtz free energy density. At equilibrium, the total Helmholtz free energy of the system with a planar interface reaches at the minimum state, so the calculation of the interfacial tension of a planar interface with the DGT in principle is a minimization problem. This minimization problem can be formulated as a set of coupled second-order differential equations by following the EulerLagrange equation. The fluid phase equilibrium compositions provide the split boundary conditions. When the geometric mean rule is used for the cross influence parameters, the set of differential equations simplifies drastically and reduces to a set of nonlinear algebraic equations. This is the geometric-mean DGT, and it is the most widely used version in many practical applications [7-11], due to its simplicity, efficiency and satisfactory performance. It will be denoted as the GM-DGT in this work hereafter.

The numerical implementation of the DGT is not trivial. It is commonly done to choose a variable that changes monotonically between the two bulk phases as the independent one in the implementation of the GM-DGT. The density of a reference component is most commonly used $[1,9,25]$, while some people recommended using a path function $[5,19,26]$, which might be still working when none of the components has a monotonic density between the two bulk phases. Usually a fixed number of points is used to discretize the domain when solving the problem $[1,5,9,25]$, which is neither flexible nor efficient. Larsen et al. [18] proposed an approach to automatically refine the density profiles during the calculation, which can significantly improve the efficiency. As pointed by Liang et al. [19], the technique for constructing the phase envelope developed by Michelsen [27,28] can be used as well, since the same structure of the equations 
can be formulated. This technique is used in some of the following calculations in this work and it is shown that it could be 5-10 times faster than the approach using 500 equidistant points to discretize the problem domain (interface width). A brief introduction of solution procedures of the GM-DGT is given in Appendix A.

The DGT in the generalized form, i.e. the cross influence parameter that deviates from the geometric mean, provides a potential alternative when the GM-DGT does not work satisfactorily. Both the finite difference technique and the finite element method are commonly used [1,5,29,30] for solving the coupled second-order differential equations. The discretization requires a finite value for the interface width, and it is common practice to use a sufficiently large value relative to the 'effective' interface width. According to previous literature [1,8,29,30], these algorithms are unstable. To improve the stability, Qiao et al. [29] and Mu et al. [30] proposed to add a timederivative term. In these practices, a fixed and relatively large number is used to discretize the problem domain. With complex models in particular the evaluations of the thermodynamic properties, e.g. the chemical potentials, are usually the time-consuming part of the calculation. Therefore, there is no doubt that unnecessarily large interface width and large number of discretization points will affect the efficiency of the algorithms. In this work, we shall further analyze the characteristics of the DGT, which gives the criterion of an adequate interface width for the interfacial tension calculations. We shall propose to use the combination of GaussLobatto quadrature and polynomial approximations for evaluating the interfacial tension from the density profiles, which makes it possible to use less discretization points. We shall solve the DGT with a given interface width by an optimization method instead of solving the coupled second-order differential equations, which are in principle equivalent, but it would be easier to extend the optimization method to solve the DGT with density dependent influence parameters.

The rest of this work is organized as follows: the analysis of the theory and the proposed algorithm are presented in Section 2; the model and parameters are briefly introduced in Section 3; the performance of the algorithm is investigated for various systems in Section 4, followed by the conclusions in Section 5. 


\section{Theory}

\subsection{Formulation of the problem}

Within the framework of the DGT, the Helmholtz free energy of a system with a planar interface consists of two contributions

$$
F=A \int_{-\infty}^{\infty}\left[\left(\sum_{i} \rho_{i} \mu_{i}-P\right)+\frac{1}{2} \sum_{i} \sum_{j} c_{i j} \frac{d \rho_{i}}{d x} \frac{d \rho_{j}}{d x}\right] d x
$$

where $F$ is the total Helmholtz free energy, $A$ is the interface area, $\rho_{i}, d \rho_{i} / d x$ and $\mu_{i}$ are the density, the density gradient and the chemical potential of component $i, P$ is the pressure, $x$ is the spatial dimension (position) and $c_{i j}$ is the cross influence parameter between molecules $i$ and $j$.

At equilibrium, the Helmholtz free energy defined in equation (1) reaches the minimum state, and the chemical potential of each component is the same everywhere in the system. With Legendre transformation, equivalently the grand potential reaches the minimum state, which turns out that the interfacial tension is also at the minimum state according to its definition. More details can be found in the PhD thesis of Cornelisse [5]. The interfacial tension can be calculated by finding the density profiles that minimize

$$
\begin{aligned}
\gamma=\int_{-\infty}^{\infty}\left\{\left[\sum_{i} \rho_{i}\left(\mu_{i}-\mu_{i 0}\right)-\left(P-P_{0}\right)\right]+\frac{1}{2} \sum_{i} \sum_{j} c_{i j} \frac{d \rho_{i}}{d x} \frac{d \rho_{j}}{d x}\right\} d x \\
=\int_{-\infty}^{\infty}\left\{\left[\overline{\boldsymbol{\rho}}^{T}\left(\overline{\boldsymbol{\mu}}-\overline{\boldsymbol{\mu}}_{0}\right)-\left(P-P_{0}\right)\right]+\frac{1}{2}\left(\frac{d \overline{\boldsymbol{\rho}}}{d x}\right)^{T} \underline{\boldsymbol{c}}\left(\frac{d \overline{\boldsymbol{\rho}}}{d x}\right)\right\} d x \\
=\int_{-\infty}^{\infty}\left[t(\overline{\boldsymbol{\rho}})+g\left(\overline{\boldsymbol{\rho}}, \overline{\boldsymbol{\rho}}_{x}\right)\right] d x=\int_{-\infty}^{\infty}[t(x)+g(x)] d x
\end{aligned}
$$

where $\gamma$ is the interfacial tension. $\overline{\boldsymbol{\rho}}, \overline{\boldsymbol{\rho}}_{x}$ and $\overline{\boldsymbol{\mu}}$ are vectors of the density, the density gradient and the chemical potential of components. The subscript 0 denotes the corresponding properties of the bulk phases. $\underline{\boldsymbol{c}}$ is the influence parameter matrix. $t(\overline{\boldsymbol{\rho}})$ represents the density based tangent plane distance function, i.e. the energy term, while $g\left(\overline{\boldsymbol{\rho}}, \overline{\boldsymbol{\rho}}_{x}\right)$ describes the gradient term. Both terms are functions of the spatial dimension $x$, as denoted by the last expression. 
In general, the cross influence parameter $c_{i j}$, which may depend on temperature and/or density, is obtained from the influence parameters of pure fluids by the equation

$$
c_{i j}=\left(1-\beta_{i j}\right) \sqrt{c_{i} c_{j}}
$$

where $\beta_{i j}$ is an adjustable parameter.

The density profiles satisfy

$$
\overline{\boldsymbol{\rho}} \rightarrow \overline{\boldsymbol{\rho}}_{v}(x \rightarrow-\infty) ; \overline{\boldsymbol{\rho}} \rightarrow \overline{\boldsymbol{\rho}}_{l}(x \rightarrow+\infty)
$$

where $\overline{\boldsymbol{\rho}}_{v}$ and $\overline{\boldsymbol{\rho}}_{l}$ are the bulk phase densities, and they fulfill the equilibrium conditions

$$
\overline{\boldsymbol{\mu}}\left(\overline{\boldsymbol{\rho}}_{v}\right)=\overline{\boldsymbol{\mu}}\left(\overline{\boldsymbol{\rho}}_{l}\right)=\overline{\boldsymbol{\mu}}_{0} ; P\left(\overline{\boldsymbol{\rho}}_{v}\right)=P\left(\overline{\boldsymbol{\rho}}_{l}\right)=P_{0}
$$

In order to calculate the interfacial tension, the first step is to obtain the density profiles, which is commonly solved by the Euler-Lagrange equations

$$
\left(\mu_{i}-\mu_{i 0}\right)+\frac{1}{2} \sum_{j} \sum_{l} \frac{\partial c_{j l}}{\partial \rho_{i}} \frac{d \rho_{j}}{d x} \frac{d \rho_{l}}{d x}-\frac{\partial}{\partial x}\left(\sum_{j} c_{i j} \frac{d \rho_{j}}{d x}\right)=0
$$

Multiplying the equation (6) by $d \rho_{i} / d x$ and making the summation of all $i$, we obtain

$$
\sum_{i} \frac{d \rho_{i}}{d x}\left(\mu_{i}-\mu_{i 0}\right)-\frac{1}{2} \sum_{i} \sum_{j} \frac{\partial c_{i j}}{\partial x} \frac{d \rho_{i}}{d x} \frac{d \rho_{j}}{d x}-\sum_{i} \sum_{j} c_{i j} \frac{d \rho_{i}}{d x} \frac{d^{2} \rho_{j}}{d x^{2}}=0
$$

which gives

$$
\frac{d g\left(\overline{\boldsymbol{\rho}}, \overline{\boldsymbol{\rho}}_{x}\right)}{d x}=\frac{1}{2} \frac{d}{d x}\left(\sum_{i} \sum_{j} c_{i j} \frac{d \rho_{i}}{d x} \frac{d \rho_{j}}{d x}\right)=\sum_{i} \frac{d \rho_{i}}{d x}\left(\mu_{i}-\mu_{i 0}\right)=\frac{d t(\overline{\boldsymbol{\rho}})}{d x}
$$

and therefore

$$
g(x)-t(x)=\text { constant }
$$

Since $g(x)$ and $t(x)$ both vanish at infinity, the constant becomes zero, resulting in $g(x)=t(x)$, as shown in the $\mathrm{PhD}$ thesis of Cornelisse (equation 2.58) [5]. This relationship is universal 
irrespective of whether the influence parameter is density dependent or not. In this work, however, we will focus on solving cases with a constant $\beta_{i j}$. With this assumption, the set of equation (6) is transformed into a set of coupled second-order differential equations

$$
\sum_{j} c_{i j} \frac{d^{2} \rho_{j}}{d x^{2}}=\mu_{i}-\mu_{i 0}
$$

There are some approaches available for solving this set of differential equations. One approach is a coordinate transformation, e.g. $\tanh (x)$, which allows one to work in a finite interval. As discussed by Miqueu et al. [8], this approach may lead to infinite number of solutions and it is difficult to be generalized to multicomponent systems. Another approach is to replace the spatial variable $x$ by a so-called path function. For example, the density of one component as the reference variable had been used in some works, e.g. Carey [1] and Miqueu et al. [8] More discussions on using a path function can be found in the $\mathrm{PhD}$ thesis of Cornelisse [5].

An alternative is to replace the infinite interval with a finite interval, which is a simpler and more straightforward approach. However, it was considered unstable and sensitive to the choice of the finite interval [1, 8, 29, 30]. Qiao et al. [29] and $\mathrm{Mu}$ et al. [30] proposed to add a time-derivative term to stabilize the algorithm. In this work, we shall mathematically show a criterion for an adequate finite interval. The derivation process will also lead to an efficient and robust algorithm without adding any term, by using linear density profiles as the initial estimates.

For a finite interval, we replace the original problem by minimizing the following equation with respect to the density profiles

$$
\gamma(L)=\int_{0}^{L}\left[t(\overline{\boldsymbol{\rho}})+g\left(\overline{\boldsymbol{\rho}}, \overline{\boldsymbol{\rho}}_{x}\right)\right] d x=I_{t}+I_{g}
$$

with

$$
\begin{gathered}
\overline{\boldsymbol{\rho}}(0) \rightarrow \overline{\boldsymbol{\rho}}_{v} ; \overline{\boldsymbol{\rho}}(L) \rightarrow \overline{\boldsymbol{\rho}}_{l} \\
I_{t}=\int_{0}^{L} t(x) d x ; I_{g}=\int_{0}^{L} g(x) d x
\end{gathered}
$$


where $I_{t}$ is the integral of the energy term, $I_{g}$ is the integral of the gradient term, and $L$ is the interface width. When $L$ is large enough, we may expect $\gamma(L)$ to approach the correct one $\gamma(\infty)$.

The Euler-Lagrange equations result in exactly the same set of differential equations as previously, i.e. equation (6) and equation (10), but now with boundary conditions at $x=0$ and $x=L$. Hence, equation (9) becomes

$$
g(x)-t(x)=g(0)=g(L)
$$

This is because $t(0)=t(L)=0$, as the equilibrium phases are trivial solutions for the tangent plane distance function. This reveals that: (i) the difference of these two terms is constant, which is equal to the value of the gradient term at the two end points; and (ii) the gradient term is always larger than the energy term. By integrating equation (13), we obtain

$$
\int_{0}^{L} g(x) d x-\int_{0}^{L} t(x) d x=I_{g}-I_{t}=L \times g(0)=L \times g(L)
$$

\subsection{Criterion for an adequate interface width $L$}

Introduce the transformation $x=L z$, and let

$$
\begin{gathered}
T(z)=t(\overline{\boldsymbol{\rho}}(z))=t(\overline{\boldsymbol{\rho}}(x))=t(x) \\
G(z)=G\left(\overline{\boldsymbol{\rho}}, \overline{\boldsymbol{\rho}}_{z}\right)=\frac{1}{2}\left(\frac{d \overline{\boldsymbol{\rho}}}{d z}\right)^{T} \underline{\boldsymbol{c}}\left(\frac{d \overline{\boldsymbol{\rho}}}{d z}\right)=L^{2} g(x)
\end{gathered}
$$

Equation (11) can be rewritten as

$$
\gamma(L)=L \int_{0}^{1} t[\overline{\boldsymbol{\rho}}(z)] d z+\frac{1}{L} \int_{0}^{1} \frac{1}{2}\left(\frac{d \overline{\boldsymbol{\rho}}}{d z}\right)^{T} \underline{\boldsymbol{c}}\left(\frac{d \overline{\boldsymbol{\rho}}}{d z}\right) d z=L \int_{0}^{1} T(z) d z+\frac{1}{L} \int_{0}^{1} G(z) d z
$$

Let

$$
i_{t}=\int_{0}^{1} T(z) d z ; i_{g}=\int_{0}^{1} G(z) d z
$$


we have,

$$
\gamma(L)=L i_{t}+\frac{1}{L} i_{g}
$$

If differentiating equation (19) with respect to $L$, we obtain

$$
\frac{\partial \gamma}{\partial L}=\left(i_{t}-\frac{1}{L^{2}} i_{g}\right)+\left(L \frac{\partial i_{t}}{\partial L}+\frac{1}{L} \frac{\partial i_{g}}{\partial L}\right)
$$

The terms in the first parentheses come from the fact as $\gamma$ is an explicit function of $L$, while the terms in the second parentheses are the derivatives via density profiles and the summation is exactly zero, as the density profiles are determined to minimize the integral. By multiplying $L$ on both sides of equation (20), we obtain

$$
L \frac{\partial \gamma}{\partial L}=L i_{t}-\frac{1}{L} i_{g}=I_{t}-I_{g}
$$

By comparing this relationship to equation (14), it leads to

$$
L \frac{\partial \gamma}{\partial L}=-L \times g(0)=-L \times g(L) \leftrightarrow \frac{\partial \gamma}{\partial L}=-g(0)=-g(L)
$$

As expected, it is proven here that the interfacial tension decreases as the interface width $L$ increases, and the derivative in equation (22) approaches to zero as $L$ goes to infinity. Provided this derivative is small, $\gamma(L)$ is an adequate representation of the exact interfacial tension $\gamma(\infty)$, and a larger $L$ is needed if this derivative is too large, which naturally becomes a criterion for an adequate interface width $\mathrm{L}$ in the interfacial tension calculations.

\subsection{Numerical solution approach for a given interface width $L$}

A combination of Gauss-Lobatto quadrature and polynomial approximation based on Lagrange interpolation is used to evaluate the integrals

$$
\int_{0}^{1} y(z) d z \approx \sum_{k=0}^{N+1} w_{k} y\left(z_{k}\right)
$$


where $z_{0}=0, z_{N+1}=1$ and $z_{k}, k=1,2, \cdots, N$ are the zeros of the $\mathrm{N}^{\text {th }}$ degree Jacobi polynomial $P_{N}^{(1,1)}(z)$. The $w_{k}$ is the corresponding quadrature weight of quadrature point $k$. The quadrature is exact provided $y(z)$ is a polynomial of degree less than $2 N+2$. These Jacobi polynomials satisfy the orthogonality relations

$$
\int_{0}^{1} z(z-1) P_{N}^{(1,1)}(z) z^{k} d z=0 \quad(k=0,1,2, \cdots, N-1)
$$

The density profiles are approximated by

$$
\overline{\boldsymbol{\rho}}(z) \approx \sum_{k=0}^{N+1} \overline{\boldsymbol{\rho}}\left(z_{k}\right) \mathcal{L}_{k}(z)
$$

where $\mathcal{L}_{k}(z)$ are the Lagrange polynomials, given by

$$
\begin{aligned}
\mathcal{L}_{k}(z) & =\frac{p(z)}{\left(z-z_{k}\right) p^{\prime}\left(z_{k}\right)} \\
p(z) & =\prod_{m=0}^{N+1}\left(z-z_{m}\right)
\end{aligned}
$$

With these expressions we obtain

$$
\frac{d \overline{\boldsymbol{\rho}}(z)}{d z}=\sum_{k=0}^{N+1} \rho\left(z_{k}\right) \frac{d \mathcal{L}_{k}(z)}{d z}
$$

and, in particular, at the quadrature points,

$$
\left(\frac{d \boldsymbol{\rho}(z)}{d z}\right)_{z_{m}}=\sum_{k=0}^{N+1} \rho\left(z_{k}\right)\left(\frac{d \mathcal{L}_{k}(z)}{d z}\right)_{z_{m}}=\sum_{k=0}^{N+1} A_{m k} \rho\left(z_{k}\right)
$$

where

$$
A_{m k}=\left(\frac{d \mathcal{L}_{k}(z)}{d z}\right)_{z_{m}}
$$


Procedures and computer source code for calculating the quadrature points, quadrature weights and the relevant coefficient matrices can be found in the book of Villadsen and Michelsen [31].

With this information available, we can calculate the integral of the gradient term

$$
i_{g}=\frac{1}{2} \sum_{i}^{C} \sum_{j}^{C} c_{i j} \sum_{k=0}^{N+1} w_{k}\left(\sum_{m=0}^{N+1} A_{k m} \rho_{i, m}\right)\left(\sum_{n=0}^{N+1} A_{k n} \rho_{j, n}\right)
$$

where $\rho_{i, m}$ and $\rho_{j, n}$ are, respectively, the densities of component $i$ at quadrature point $m$ and the density of component $j$ at quadrature point $n$. $C$ is the total number of components.

The derivatives of $i_{g}$ with respect to densities are given by

$$
\begin{gathered}
\frac{\partial i_{g}}{\partial \rho_{i, m}}=\sum_{j}^{C} c_{i j} \sum_{k=0}^{N+1} w_{k} A_{k m}\left(\sum_{n=0}^{N+1} A_{k n} \rho_{j, n}\right) \\
\frac{\partial^{2} i_{g}}{\partial \rho_{i, m} \partial \rho_{j, n}}=c_{i j}\left(\sum_{k=0}^{N+1} w_{k} A_{k m} A_{k n}\right)
\end{gathered}
$$

We note that the matrix inside the parentheses of the second-order derivatives does not depend on density and can be calculated as long as the number of quadrature points is determined.

The integral of the energy term is calculable

$$
i_{t}=\sum_{k=0}^{N+1} w_{k} t\left(\overline{\boldsymbol{\rho}}_{k}\right)=\sum_{k=1}^{N} w_{k} t\left(\overline{\boldsymbol{\rho}}_{k}\right)
$$

since $t(x)$ is zero at the endpoints as mentioned above. The derivatives are

$$
\begin{gathered}
\frac{\partial i_{t}}{\partial \rho_{i, m}}=\mu_{i}\left(\overline{\boldsymbol{\rho}}_{m}\right)-\mu_{i 0} \\
\frac{\partial^{2} i_{t}}{\partial \rho_{i, m} \partial \rho_{j, n}}=\delta_{m n} \frac{\partial \mu_{i}\left(\overline{\boldsymbol{\rho}}_{m}\right)}{\partial \rho_{j}}
\end{gathered}
$$


where $\overline{\boldsymbol{\rho}}_{m}$ are the densities of all components at the quadrature point $m . \delta_{m n}$ is the Kronecker delta function of the two quadrature points $m$ and $n$.

The condition for optimality is that the total gradient of the function must equal zero, i.e.

$$
\frac{\partial \gamma}{\partial \rho_{i, m}}=L \frac{\partial i_{t}}{\partial \rho_{i, m}}+\frac{1}{L} \frac{\partial i_{g}}{\partial \rho_{i, m}}=0
$$

We reorganize the expression and use a vector $\overline{\boldsymbol{f}}$ to represent the total gradient, since the letters $g$ and $G$ have been used for the gradient term in the theory.

$$
\overline{\boldsymbol{f}}=\left[L^{2} \frac{\partial i_{t}}{\partial \rho_{i, m}}+\frac{\partial i_{g}}{\partial \rho_{i, m}}\right]_{N \times C}=0
$$

There is a total of $N \times C$ unknowns, i.e. the densities of $\mathrm{C}$ components at $\mathrm{N}$ quadrature points. These unknowns are found by the restricted step algorithm [32], which is globally and quadratically convergent to at least a local minimum. The required Hessian matrix is calculated from the second-order derivatives

$$
\underline{\boldsymbol{H}}=\left[\frac{\partial^{2} \gamma}{\partial \rho_{i, m} \partial \rho_{j, n}}\right]_{N \times C, N \times C}=\left[L^{2} \frac{\partial^{2} i_{t}}{\partial \rho_{i, m} \partial \rho_{j, n}}+\frac{\partial^{2} i_{g}}{\partial \rho_{i, m} \partial \rho_{j, n}}\right]_{N \times C, N \times C}
$$

The update at each iterative step is found by

$$
\Delta \overline{\boldsymbol{\rho}}=-(\underline{\boldsymbol{H}}+\lambda \underline{\boldsymbol{I}})^{-1} \times \overline{\boldsymbol{f}}
$$

where $\lambda$ is a correction constant when $\underline{\boldsymbol{H}}$ is not positive definite or when the step does not decrease the objective function. More details on this optimization method can be found in the book of Fletcher [32].

\subsection{The entire algorithm}

According to the analysis presented above, it is natural for us to propose an inner-outer two loop algorithm to calculate the interfacial tension within the framework of the DGT. The outer loop deals with the relationship between the interfacial tension and the interface width, presented in Section 2.2. If the integral of the energy term and the integral of the gradient term are close enough, i.e. $\left(I_{g}-I_{t}<\tau I_{t}\right)$, we stop the entire algorithm and obtain the interfacial tension. The 
inner loop minimizes the interfacial tension for a given interface width by adjusting the density profiles, presented in Section 2.3. The entire algorithm is described in Table 1. Some details on the strategies for initial estimates and updating the interface width L, a better approximation of the interfacial tension without additional cost and a further comment on the inner loop solver are discussed below.

\subsubsection{Strategies for initial estimates and updating the interface width $L$}

It is readily known from equation (13) and equation (22) that the integral of the energy term and the integral of the gradient term, respectively, increases and decreases as the interface width increases. This tells that the gradient term dominates when the interface width $L$ is small enough, which is the basis to use the linear density profiles as the initial estimates to start the entire algorithm. With a fixed update for the interface width, e.g. $0.15 \mathrm{~nm}$, the algorithm should be robust enough by using the converged density profiles from the current interface width as the initial estimates for the next interface width, i.e. the next iteration step in the outer loop. However, the efficiency can be further improved.

If differentiating equation (38) with respect to the interface width $L$, we obtain

$$
\underline{\boldsymbol{H}} \frac{d \overline{\boldsymbol{\rho}}}{d L}=-2 L\left(\overline{\boldsymbol{\mu}}-\overline{\boldsymbol{\mu}}_{0}\right)
$$

The decomposition of the Jacobian matrix $\underline{\boldsymbol{H}}$ has already been available from equation (40), so the density profiles at a new $L$ can be calculated from

$$
\overline{\boldsymbol{\rho}}\left(L^{\text {new }}\right)=\overline{\boldsymbol{\rho}}\left(L^{\text {old }}\right)+\left(L^{\text {new }}-L^{\text {old }}\right) \frac{d \overline{\boldsymbol{\rho}}}{d L}=\overline{\boldsymbol{\rho}}\left(L^{\text {old }}\right)+d L \frac{d \overline{\boldsymbol{\rho}}}{d L}
$$

The derivatives $d \overline{\boldsymbol{\rho}} / d L$ are the sensitivity of the density profiles with respect to the interface width. This is the step S6.5 in the algorithm.

It is common practice to adaptively update the independent parameter in the phase envelope technique [27,28]. Therefore, starting with $d L=0.15 \mathrm{~nm}$, the following procedure is used to update $d L$ : if the inner loop converges with 1 or 2 steps, $d L=1.5 \times d L$; if the inner loop uses 3 steps, $d L=1.2 \times d L$; if the inner loop needs more than 6 steps, $d L=0.5 \times d L$; otherwise, $d L$ is kept no change, which is the step S6.3 in the algorithm. 


\subsubsection{A better approximation for the interfacial tension}

Without additional cost, the density profiles $\overline{\boldsymbol{\rho}}(z)$ available during the iterative process can be used to create an approximation superior to $\gamma(L)$ to the exact value. By considering $\overline{\boldsymbol{\rho}}(z)$ as a parametric representation of the density profiles, we can transform equation (11) to

$$
\gamma(L)=\int_{0}^{L}\left[t(\overline{\boldsymbol{\rho}})+G\left(\overline{\boldsymbol{\rho}}, \overline{\boldsymbol{\rho}}_{z}\right)\left(\frac{d z}{d x}\right)^{2}\right] \frac{d x}{d z} d z=\int_{0}^{1}\left[t(\overline{\boldsymbol{\rho}}) \frac{d x}{d z}+G\left(\overline{\boldsymbol{\rho}}, \overline{\boldsymbol{\rho}}_{z}\right) \frac{d z}{d x}\right] d z
$$

It needs to point out that the relation $x=L z$ does not hold anymore in this transformation. The integrand in equation (43) is minimized everywhere, provided we choose:

$$
\frac{d x}{d z}=\sqrt{\frac{G\left(\overline{\boldsymbol{\rho}}, \overline{\boldsymbol{\rho}}_{z}\right)}{t(\overline{\boldsymbol{\rho}})}}
$$

This results in:

$$
\gamma_{1}(L)=2 \int_{0}^{1} \sqrt{t(\overline{\boldsymbol{\rho}}) G\left(\overline{\boldsymbol{\rho}}, \overline{\boldsymbol{\rho}}_{z}\right)} d z
$$

\subsubsection{A further comment on inner loop solver}

In this work, we use the direct optimization approach in the inner loop. It is possible to replace the optimization problem by solving the set of the coupled second-order differential equations, i.e. equation (10), in the inner loop. If the coupled second-order differential equations are solved by the collocation method combined with the Gaussian quadrature for the integrals [31], it is possible to get a similar efficiency. However, we prefer to use the optimization approach, since it guarantees global convergence (not the same as converging to the global minimum). More importantly, it is more straightforward to extend the algorithm to density dependent (cross) influence parameters. 
Table 1. The entire algorithm

Step S1: Choose a model and setup relevant parameters, e.g. EOS parameters, binary interaction parameters, influence parameters, adjustable parameter $\beta_{i j}$ for the cross influence parameter, etc. Step S2: Specify the conditions (T, P, composition), and obtain the properties of bulk phases $\left(\overline{\boldsymbol{\rho}}_{\boldsymbol{v}}\right.$, $\overline{\boldsymbol{\rho}}_{l}, \overline{\boldsymbol{\mu}}_{0}$ and $\left.P_{0}\right)$. If not available, make phase equilibrium calculations to get them.

Step S3: Choose the number of quadrature points $N$ and calculate the point positions $\left\{z_{k}\right\}$, the density independent derivative matrices, and the quadrature weights $\left\{w_{k}\right\}$, for which the source code are available from the book of Villadsen and Michelsen [31].

Step S4: Setup solver related parameters, i.e. convergence criteria for the inner and outer loops, the (initial) change of the interface width at each step $d L$ and initial interface width $\mathcal{L}^{\text {init }}$.

Step S5: Generate the initial density profiles from $\rho_{i, k}=\rho_{i, v}+z_{k}\left(\rho_{i, l}-\rho_{i, v}\right)\left(z_{k}\right.$ is the position of quadrature point $k ; \rho_{i, v}$ and $\rho_{i, l}$ are the vapor and liquid densities of component $i$ ).

Step S6 (outer loop): Count the iteration number, and if it exceeds a given maximum value (e.g. 250), go back step S3 or S4 to restart the program; and set inner loop iteration number to zero.

Step S6.1 (inner loop): Count the iteration number.

Step S6.1.1: Calculate the derivatives of the gradient term.

Step S6.1.2: Call the thermodynamic model to calculate the chemical potential and pressure at each quadrature point in order to calculate the derivatives of the energy term.

Step S6.1.3: Evaluate the integrals $i_{t}, i_{g}$ and the one in equation (45).

Step S6.1.4: Calculate the updates of the density $\Delta \overline{\boldsymbol{\rho}}$ using the restricted step approach.

Step S6.1.5: If $|\Delta \overline{\boldsymbol{\rho}}|^{2}<e$ (inner loop convergence criterion), exit the inner loop.

Step S6.1.7: $\overline{\boldsymbol{\rho}}_{\text {inner }}^{\text {new }}=\overline{\boldsymbol{\rho}}_{\text {inner }}^{\text {old }}+\Delta \overline{\boldsymbol{\rho}}$.

Step S6.2: Calculate $I_{t}$ and $I_{g}$ from $i_{t}$ and $i_{g}$. If $I_{g}-I_{t}<\tau I_{t}$ or $\left(I_{g}-I_{t}\right)$ cannot be decreased any more, obtain the interfacial tension $\gamma(L), \gamma_{1}(L)$ and other outputs, and return successfully from the algorithm.

Step S6.3: Update $d L$ depending on the number of iterations in the inner loop (Section 2.5.1) Step S6.4: Update $L^{\text {new }}=L^{\text {old }}+d L$.

Step S6.5: Generate initial estimates for $L^{\text {new }}$ from the sensitivity of the density profiles w.r.t the interface width $L$ (see more details in the Section 2.5.1). 


\section{Models and parameters}

The tangent plane distance function and the boundary conditions are calculated from the thermodynamic model. In this work, the Peng-Robinson (PR) [33], the Cubic Plus Association (CPA), proposed by Kontogeorgis et al. [34], and the simplified Perturbed-Chain Statistical Associating Fluid Theory (PC-SAFT) EOS [35] are chosen. The details of the equations, symbols and applications of these models can be found in the monograph of Kontogeorgis and Folas [36]. The pure component parameters and non-zero binary interaction parameters are listed in Tables 2 and 3, and temperature-independent influence parameters are used in this work. The corresponding references are also given in the tables.

Table 2. The pure component parameters of relevant compounds

\begin{tabular}{|c|c|c|c|c|c|c|c|}
\hline \multirow{2}{*}{ comp. } & \multicolumn{5}{|c|}{ CPA } & \multirow{2}{*}{$\begin{array}{c}c_{i} \cdot 10^{20} \\
\left(\mathrm{~J} \cdot \mathrm{m}^{5} / \mathrm{mol}^{2}\right)\end{array}$} & \multirow{2}{*}{ Ref. } \\
\hline & $b(\mathrm{~L} / \mathrm{mol})$ & $\Gamma(\mathrm{K})$ & $c_{1}$ & $\varepsilon^{H B} / k(\mathrm{~K}) \quad \kappa^{H B} \cdot 10^{3}$ & $a s c^{*}$ & & \\
\hline water & 0.014515 & 1017.34 & 0.67359 & 2003.25 & $4 \mathrm{C}$ & 1.766 & 37,20 \\
\hline ethanol & 0.049110 & 2123.83 & 0.73690 & 2589.85 & $2 B$ & 4.988 & 38,20 \\
\hline acetone & 0.06190 & 2719.57 & 0.80023 & & & 11.99 & 39,20 \\
\hline toluene & 0.09214 & 3051.36 & 0.80370 & & & 34.83 & 40,20 \\
\hline n-hexane & 0.10789 & 2640.03 & 0.83130 & & & 40.39 & 37,20 \\
\hline \multirow{2}{*}{ comp. } & \multicolumn{5}{|c|}{ PC-SAFT } & \multirow{2}{*}{$\begin{array}{c}c_{i} \cdot 10^{20} \\
\left(\mathrm{~J} \cdot \mathrm{m}^{5} / \mathrm{mol}^{2}\right)\end{array}$} & \multirow{2}{*}{ Ref. } \\
\hline & $\sigma(\AA)$ & $\epsilon / k(\mathrm{~K})$ & length ${ }^{+}$ & $\varepsilon^{H B} / k(\mathrm{~K})$ & asc & & \\
\hline water & 2.3449 & 171.67 & 2 & 1704.06 & $4 \mathrm{C}$ & 1.31 & 41,19 \\
\hline ethanol & 3.0577 & 191.90 & 2.6351 & 2574.01 & $2 \mathrm{~B}$ & 4.92 & 42,19 \\
\hline n-hexane & 3.7983 & 236.77 & 3.0576 & & & 36.5 & 43,19 \\
\hline \multirow{2}{*}{ comp. } & \multicolumn{5}{|c|}{ PR } & \multirow{2}{*}{$\begin{array}{c}c_{i} \cdot 10^{20} \\
\left(\mathrm{~J} \cdot \mathrm{m}^{5} / \mathrm{mol}^{2}\right)\end{array}$} & \multirow{2}{*}{ Ref. } \\
\hline & $T_{c}(\mathrm{~K})$ & $P_{c}\left(10^{-5} \mathrm{~Pa}\right)$ & $\omega$ & & & & \\
\hline methane & 190.56 & 45.99 & 0.0115 & & & 1.764 & 5 \\
\hline propane & 369.83 & 42.48 & 0.1523 & & & 9.290 & 5 \\
\hline n-pentane & 469.70 & 33.70 & 0.2515 & & & 28.22 & 5 \\
\hline n-decane & 617.70 & 21.10 & 0.4923 & & & 130.45 & 5 \\
\hline n-hexadecane & 723.00 & 14.00 & 0.7174 & & & 432.45 & 5 \\
\hline
\end{tabular}

*asc means the association scheme.

${ }^{+}$Usually the length of the molecular chain is denoted by $m$ in the PC-SAFT EOS. In this work, $m$ is used for subscript in the text, so we directly use length in this table. 
Table 3. The binary interaction parameters for the investigated systems

\begin{tabular}{ccc}
\hline Binary & $k_{i j}$ & Ref. \\
\hline acetone + toluene (CPA) & 0.025 & 18 \\
ethanol + n-hexane (CPA) & 0.0081 & 19 \\
ethanol + n-hexane (PC-SAFT) & 0.0102 & 19 \\
water + ethanol (CPA) & -0.0409 & 38 \\
water + ethanol (PC-SAFT) & -0.0532 & 41 \\
methane + n-decane (PR) & 0.065 & this work \\
methane + n-hexadecane (PR) & 0.065 & this work \\
\hline
\end{tabular}

\section{Results and discussion}

\subsection{Algorithm parameters}

There are four key parameters in the proposed algorithm: the number of quadrature points $(N)$, the initial interface width $\left(L^{\text {init }}\right)$, the outer loop convergence criterion $(\tau)$ and the inner loop convergence criterion $(e)$. The outer loop convergence criterion is the tolerance for the difference between the integral of the energy term and the integral of the gradient term so as to stop the entire algorithm, while the inner loop convergence criterion is for the error at the solution of the optimization problem. We consider that $L^{\text {init }}=1.0 \mathrm{~nm}, d L=0.15 \mathrm{~nm}, \tau=0.02$ and $e=0.01$ are adequate numbers for general practical applications. The typical relationship of the calculated interfacial tension against the number of quadrature points is given in Figure 1 for two systems: ethanol + n-hexane with the PC-SAFT EOS and water + ethanol with the CPA EOS, both at 298.15K. It can be seen that the calculated interfacial tension is kept constant starting around 20 quadrature points. Therefore, $N=20$ could be recommended for general practical applications when the interfacial tension is the main objective.

The density profiles for these two systems as given in Figure 2, in which the densities are scaled by their corresponding values in the liquid phase. The density profiles can be generally considered satisfactory. However, more quadrature points are recommended when very detailed density profiles are concerned. 
With these parameters, we have not seen failures in the cases discussed below, but the algorithm might fail depending on the complexity and the conditions of the being investigated system. If the algorithm fails, these parameters can be adjusted to restart the algorithm. For example, if the algorithm fails at the first outer loop, smaller $L^{\text {init }}$ or the other initial guess approaches [44] could be tried. Otherwise, more quadrature points should be tried before changing the convergence criteria, since the mole densities, the maximum value of which is usually larger than $10^{4} \mathrm{~mol} / \mathrm{m}^{3}$, are used as the independent variables in the inner loop and we shall show that $\tau=0.02$ is adequate, especially when the improved equation (45) is used.

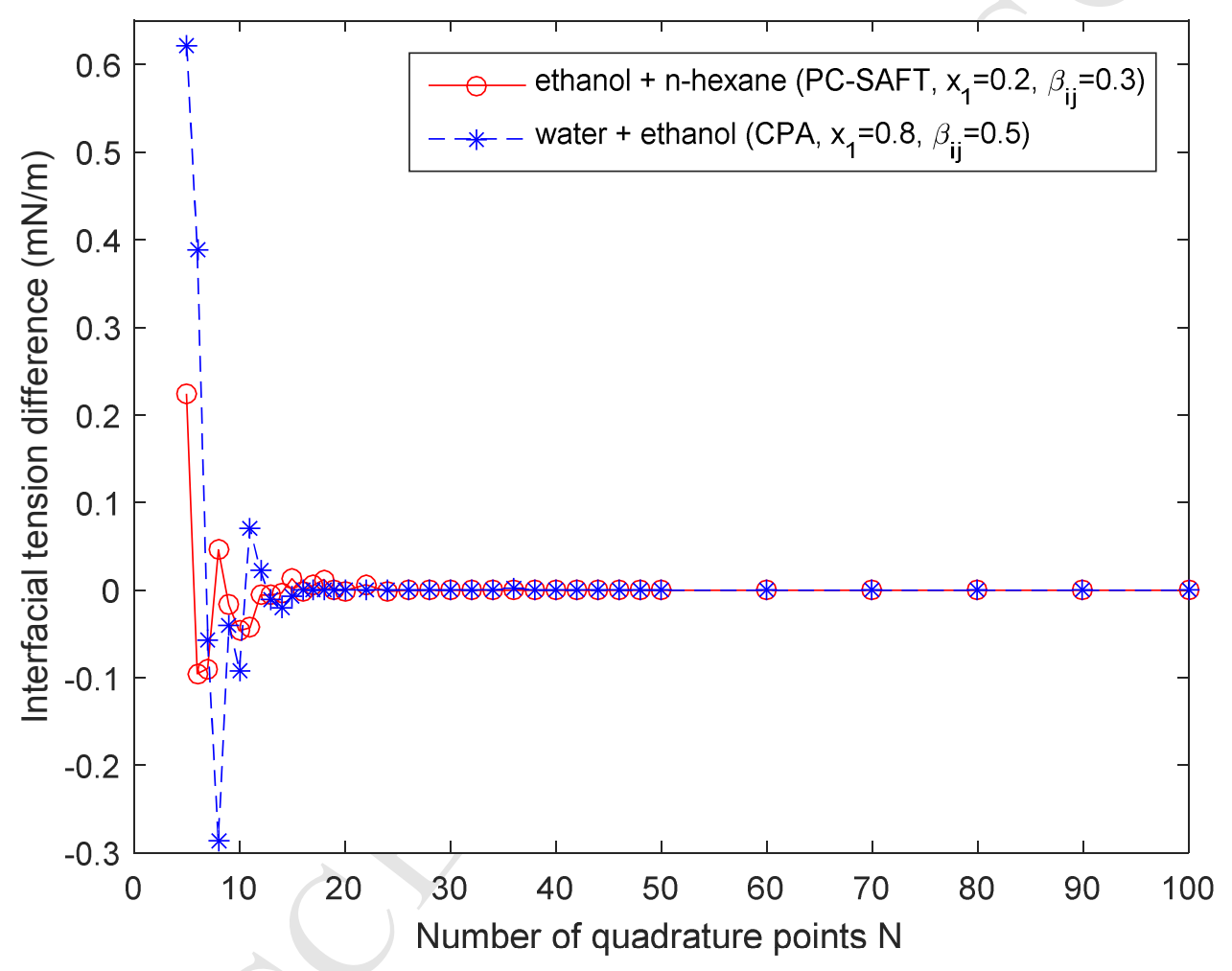

Figure 1. The calculated interfacial tension versus the number of quadrature points for ethanol + n-hexane with the PC-SAFT EOS and water + ethanol with the CPA EOS, both at 298.15K. The $y$-axis values represent the differences of the calculated interfacial tension values between $N<100$ and $N=100$. 


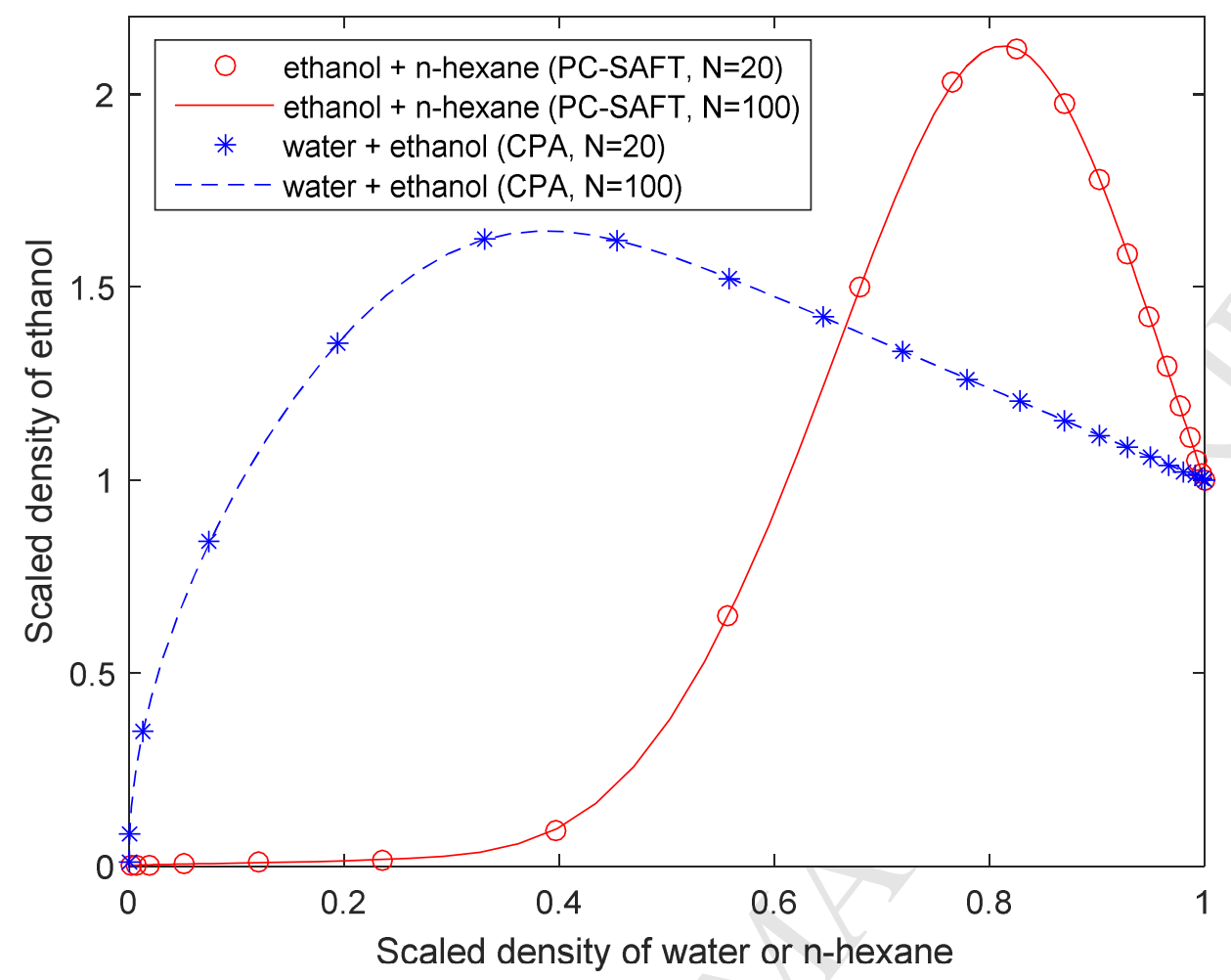

Figure 2. The scaled density profiles for ethanol + n-hexane with the PC-SAFT EOS and water + ethanol with the CPA EOS, both at $298.15 \mathrm{~K}$. The corresponding composition and adjustable parameter $\beta_{\mathrm{ij}}$ are given in Figure 1. The densities are scaled by their corresponding densities in the liquid phase. 


\subsection{Convergence behavior}

Even though we have mathematically derived the procedure in Section 2, it is still nice to show the convergence of the algorithm. The density profiles from the proposed algorithm with recommended parameters are compared in Figure 3 with those from the same algorithm with 200 quadrature points and a system size (interface width) of $5 \mathrm{~nm}$, which means that the algorithm continues until the interface width L becomes larger than $5 \mathrm{~nm}$. It can be seen from Figure 4 that the density profiles are not completely matched with each other, but the interfacial tension has converged, especially with the equation (45). A better match of the density profiles could be obtained by decreasing the outer loop convergence criterion $\tau$.

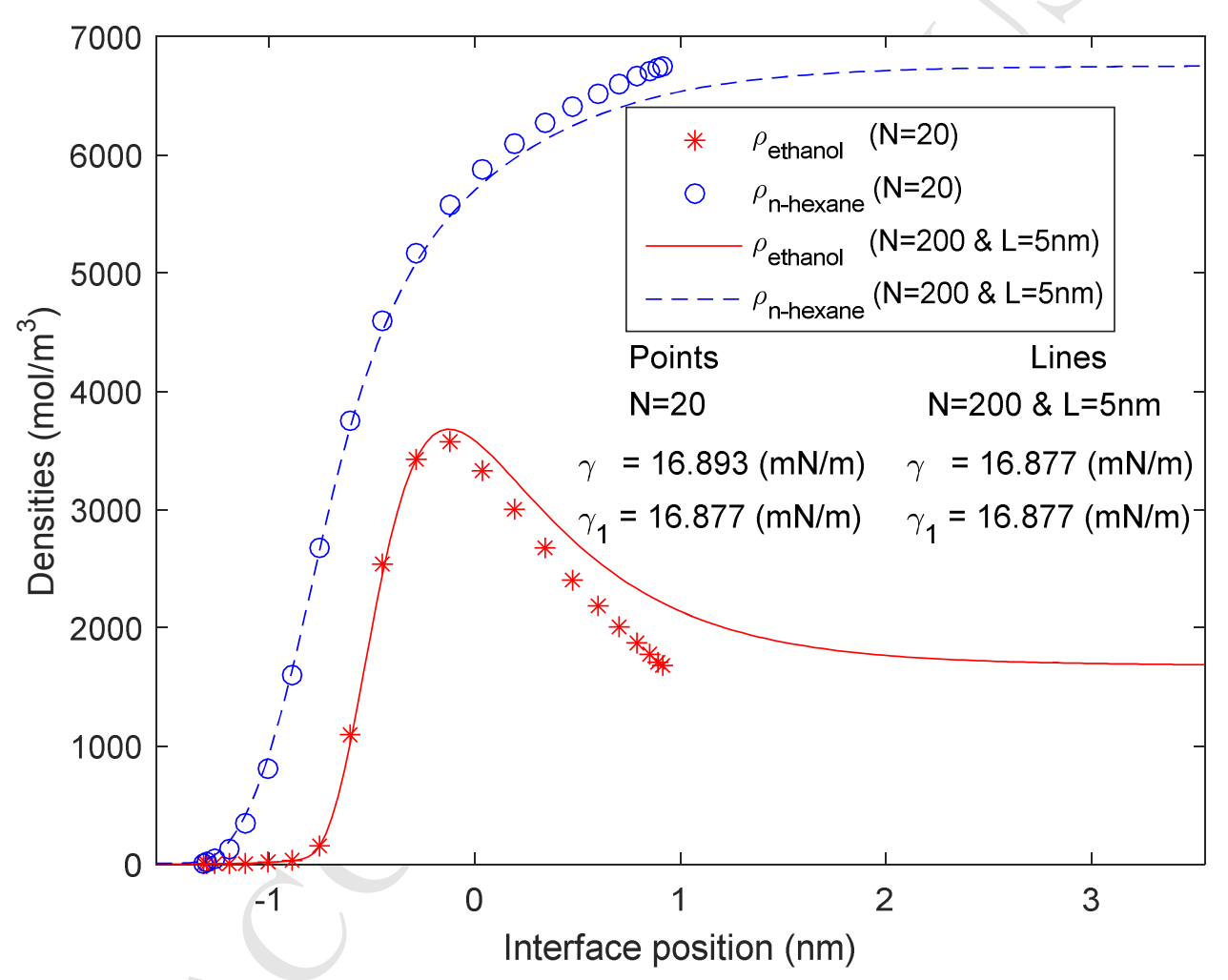

Figure 3. Density profiles of the system ethanol + n-hexane at $298.15 \mathrm{~K}$ with the PC-SAFT EOS from different approaches. The mole fraction and adjustable parameter $\beta_{i j}$ are the same as the previous figures. The points are from the standard propose algorithm, while the lines are from 200 quadrature points with a system size (interface width) of $5 \mathrm{~nm}$. The interfacial tension values from both equation (19) and equation (45) are given for these two cases. 
The calculated interfacial tensions from equation (19) and equation (45) are compared in Figure 4 for the system ethanol + n-hexane with the PC-SAFT EOS and the system water + ethanol with the CPA EOS, both at $298.15 \mathrm{~K}$. The values of the adjustable parameter $\beta_{i j}$ used here are the same as Figure 1. The results could be considered almost the same, even though a very tiny improvement has been achieved by using equation (45) to calculate the interfacial tension at some compositions in the case of ethanol $+\mathrm{n}$-hexane. This indicates that $\tau=0.02$ is adequate to be used as the convergence criterion for the outer loop. As discussed in previous works $[19,20]$, the system of ethanol $+n$-hexane is interesting and challenging for the DGT with the PC-SAFT and CPA EOS. Therefore, more discussions are given in Appendix B for this particular system.
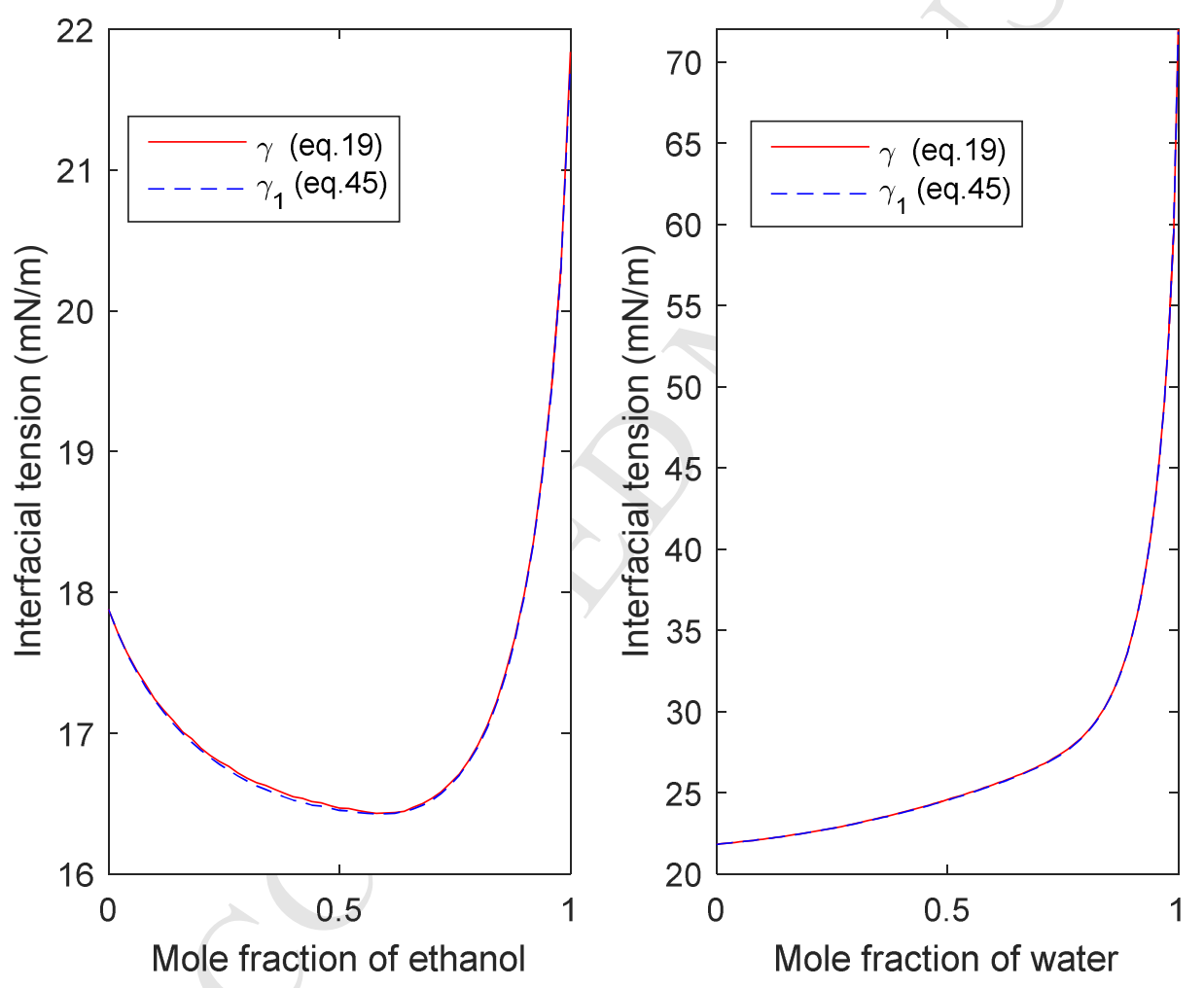

Figure 4. Interfacial tensions of ethanol $+n$-hexane with the PC-SAFT EOS and water + ethanol with the CPA EOS, both at $298.15 \mathrm{~K}$. The values of the adjustable parameters $\beta_{i j}$ are the same as the ones given in Figure 1. The red solid line and the blue dash line are the results from the proposed algorithm with the two different choices of calculating the interfacial tension, i.e. equation (19) and equation (45). 
The two ways of calculating the interfacial tension, equation (19) and equation (45), are further compared in Figure 5. The interfacial tension is found to converge much faster when the equation (45) is used. Therefore, we recommend that the equation (45) should be used in practical calculations, and it will somehow compensate for the inaccuracy, if it occurs, of the equation (19) due to the loose criterion $\tau=0.02$. It is interesting to notice that the interface widths in these two cases are smaller than $3.5 \mathrm{~nm}$, while larger values of the problem domain are usually used in other implementations [5,30].
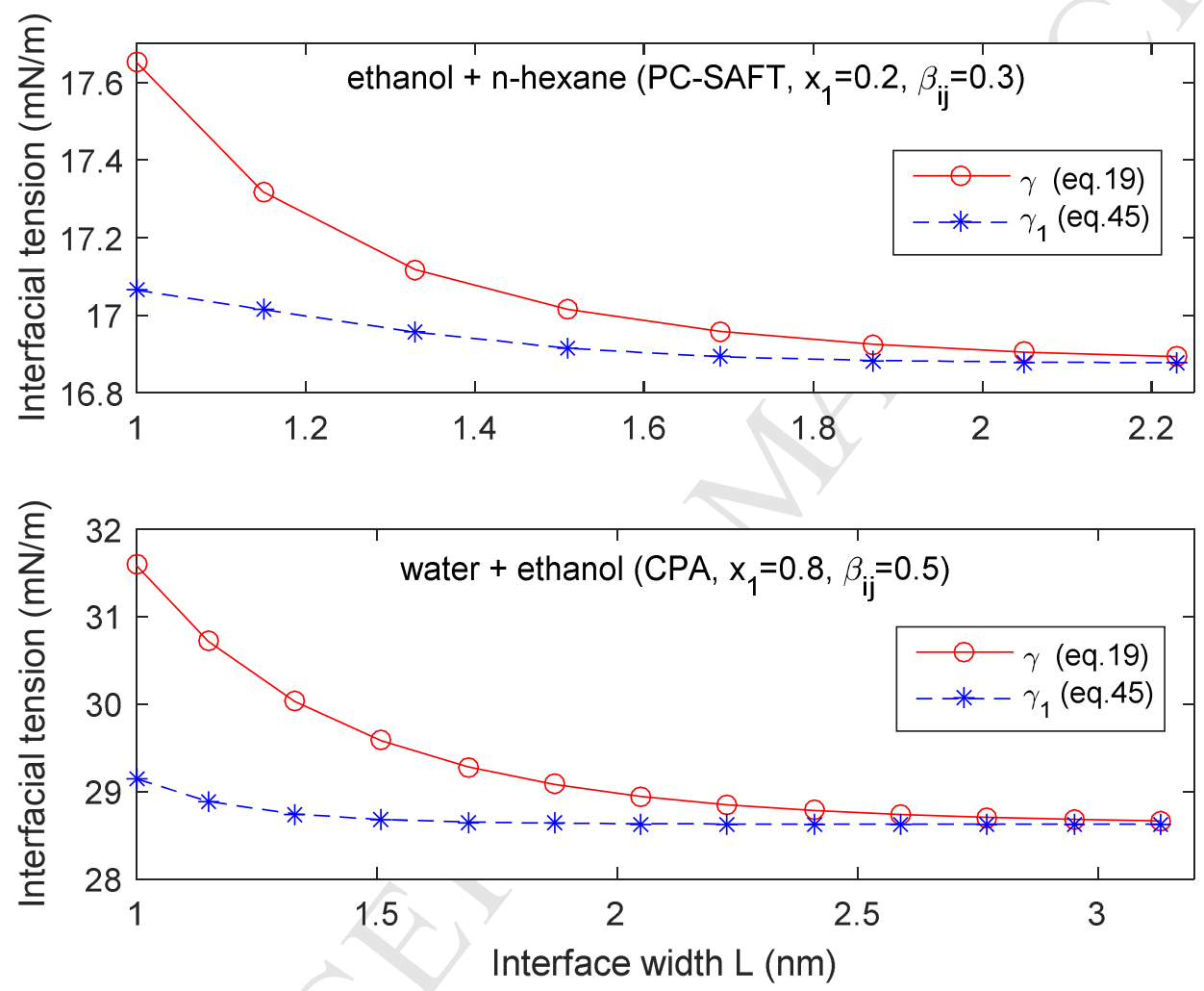

Figure 5. The calculated interfacial tension at each outer loop for the systems corresponding to those given in Figure 1. The adjustable parameter $\beta_{i j}$ and the mole fraction of the first component are given as texts in the subfigures. The two lines describe the convergence behavior of the two ways of calculating the interfacial tension, equation (19) and equation (45). 
The iteration processes of the algorithm for the same two systems are presented in Figure 6. In the figure, the number of points represents the number of iteration steps in the outer loop, while the number of iterations in the inner loop at each outer loop is the value (y-axis) of that point. Therefore, the summation of the values of all points is the total number of iterations. The total number of calling the thermodynamic model is the product of the total number of iterations and the number of quadrature points. For these two systems, the thermodynamic models are called 660 and 1160 times, respectively. This can be considered very efficiently, because no more than 3 iterations can be conducted in order to gain the same efficiency in the finite difference technique or the finite element method if 500 points are used for the discretization.

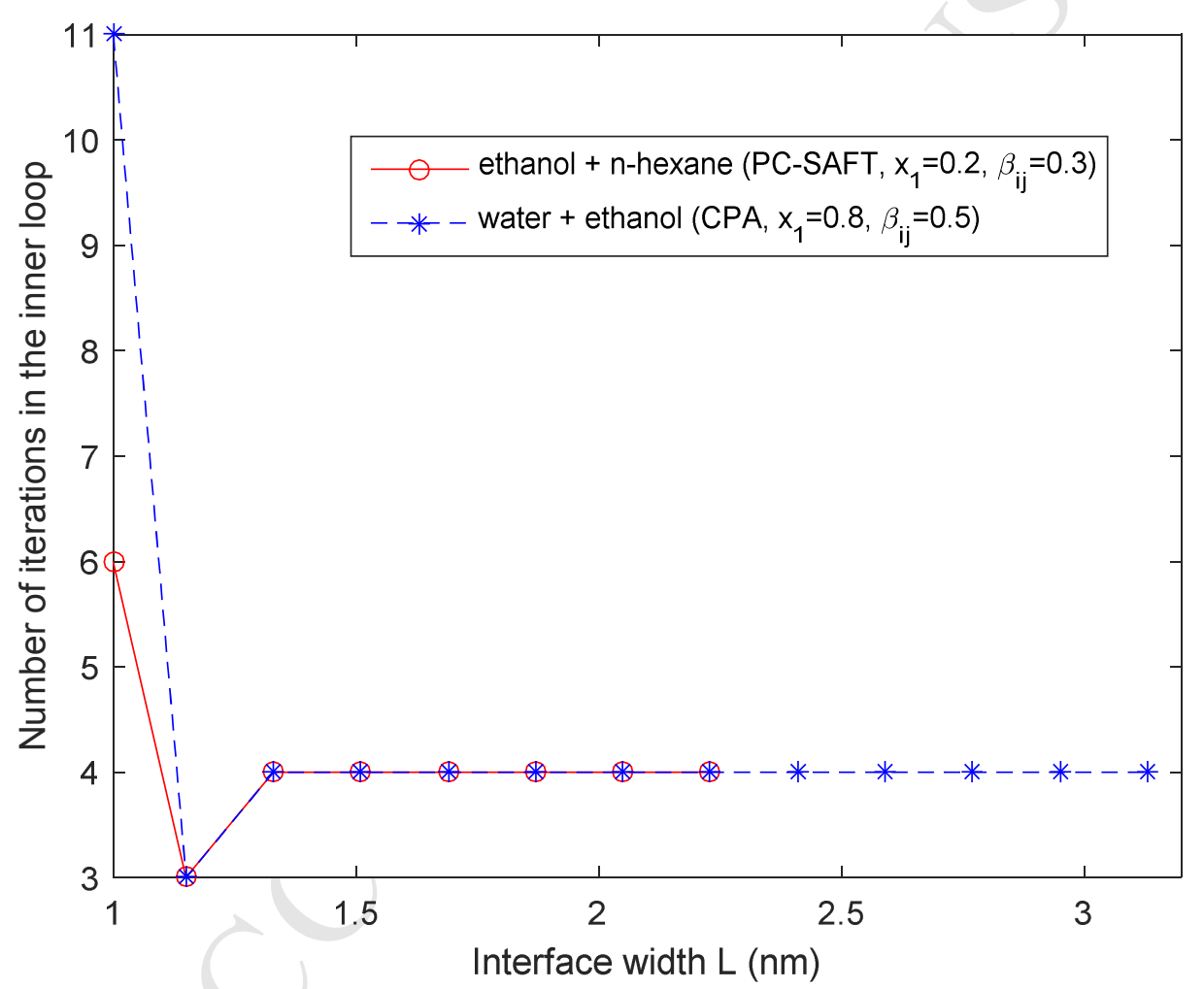

Figure 6. Iteration process of the algorithm for the systems ethanol $+\mathrm{n}$-hexane with the PCSAFT EOS and water + ethanol with the CPA EOS, both at 298.15K. The adjustable parameter $\beta_{i j}$ and the mole fraction of the first component are given in the legend. The number of points represents the number of iteration steps in the outer loop, while the number of iterations in the inner loop at each outer loop is the value (y-axis) of that point. 


\subsection{Efficiency}

The average running time of the algorithm versus the adjustable parameter $\beta_{i j}$ is presented in Figure 7 for the three systems: (1) acetone + toluene at 288.15K with the CPA EOS; (2) ethanol $+\mathrm{n}$-hexane at $298.15 \mathrm{~K}$ with the PC-SAFT EOS; (3) water + ethanol at $298.15 \mathrm{~K}$ with both the $\mathrm{CPA}$ and PC-SAFT EOS. The average running time for a given $\beta_{i j}$ is counted by

$$
\text { average running time }=\frac{\sum_{i=1}^{i=99} n r \times \text { running time }\left(x_{1}=0.01 i\right)}{n r \times 99}
$$

where $n r$ is the number of repetition for each calculation. In this case, $n r=10$, which means that the calculation is repeated 10 times for each point, and the composition is screened from 0.01 to 0.99 with a step 0.01 for the first component in the system. It has to be pointed out that mathematically $\beta_{i j}$ can be larger than 1 , as discussed by Cornelisse [5]. To the best of our knowledge, however, $\beta_{i j} \geq 1$ has not been found in reality. Therefore, $0 \leq \beta_{i j} \leq 0.99$ is taken.

The code has been compiled with the Intel Visual Fortran Composer XE 2011 and run with the processor Intel® Core ${ }^{\mathrm{TM}}$ i5-5300U CPU @ $2.30 \mathrm{GHz}$ in a laptop. It can be readily seen that the running time of all the systems is less than $0.007 \mathrm{~s}$ for the calculation of one point. The running time increases as the adjustable parameter $\beta_{i j}$ becomes larger. A high $\beta_{i j}$ could make the algorithm one time slower than a small $\beta_{i j}$, e.g. for the systems of acetone + toluene and ethanol $+n$-hexane. Figure 7 also shows that it takes longer time to calculate the interfacial tension of the system of water + ethanol compared to the system of acetone + toluene, for which the running time is less than $0.001 \mathrm{~s}$ for most situations, because there are respectively two and no associating fluids in these two systems. It can be seen that the running time with the PC-SAFT EOS is slightly longer than that with the CPA EOS for the system water + ethanol. These results reveal that the running time also depends on the complexity of system and the thermodynamic model. 


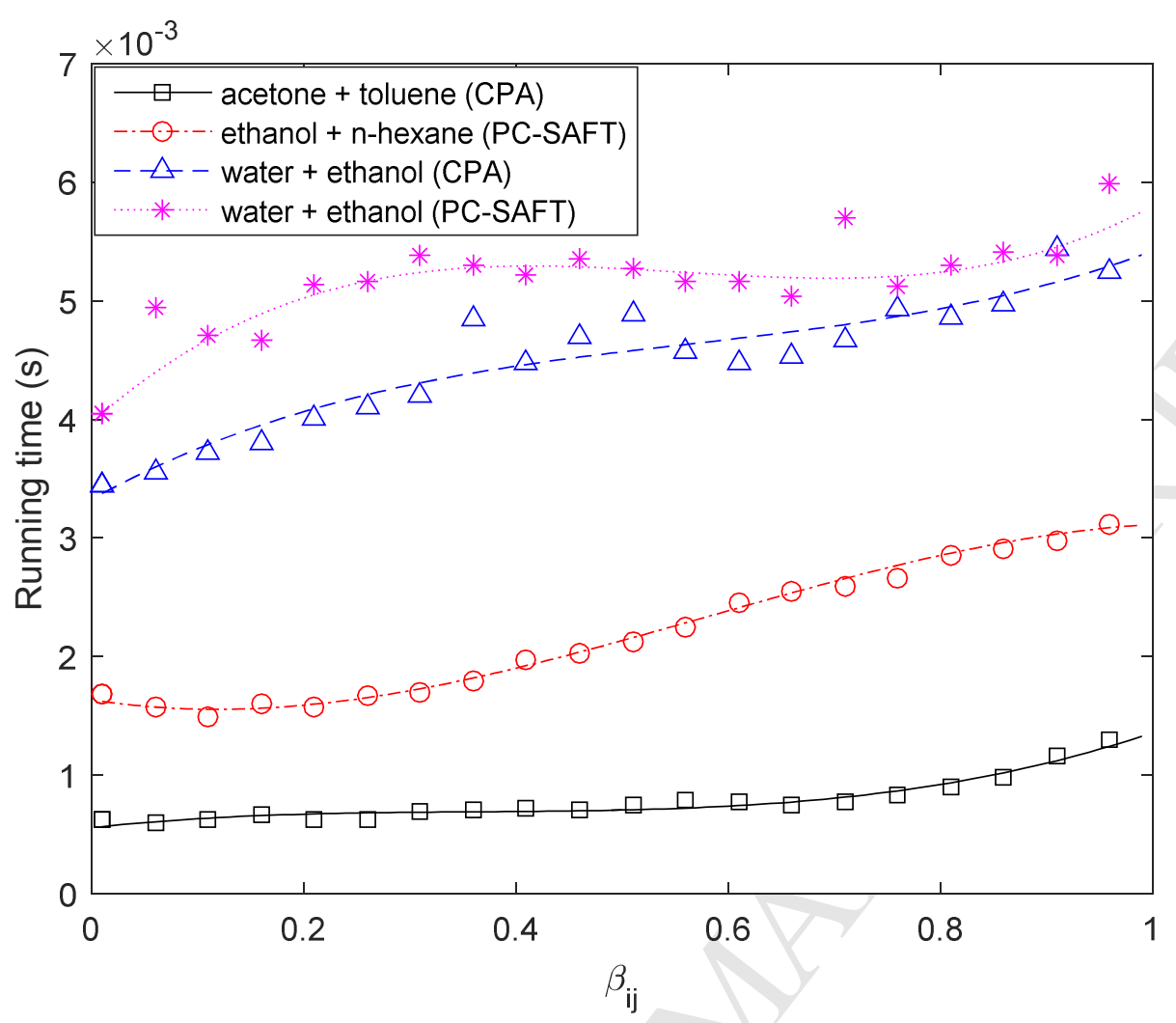

Figure 7. The average running time of the algorithm versus the adjustable parameter $\beta_{i j}$ for three systems acetone + toluene at $288.15 \mathrm{~K}$ with the CPA EOS, ethanol + n-hexane at $298.15 \mathrm{~K}$ with the PC-SAFT EOS, water + ethanol at $298.15 \mathrm{~K}$ with both the CPA and PC-SAFT EOS. The calculation method of the average running time and the machine information of the computer can be found in the text. 
As aforementioned, more quadrature points are needed if detailed density profiles are concerned. The relation of the running time and the number of quadrature points has been investigated for the same systems, as presented in Figure 8. It reveals that the running time is significantly increased as more quadrature points are used. For example, it takes 20-30 times longer when 100 quadrature points are used instead of 20 points. However, it can still be considered as an efficient algorithm, since it takes less than 0.15 s for one point calculation for all the systems.

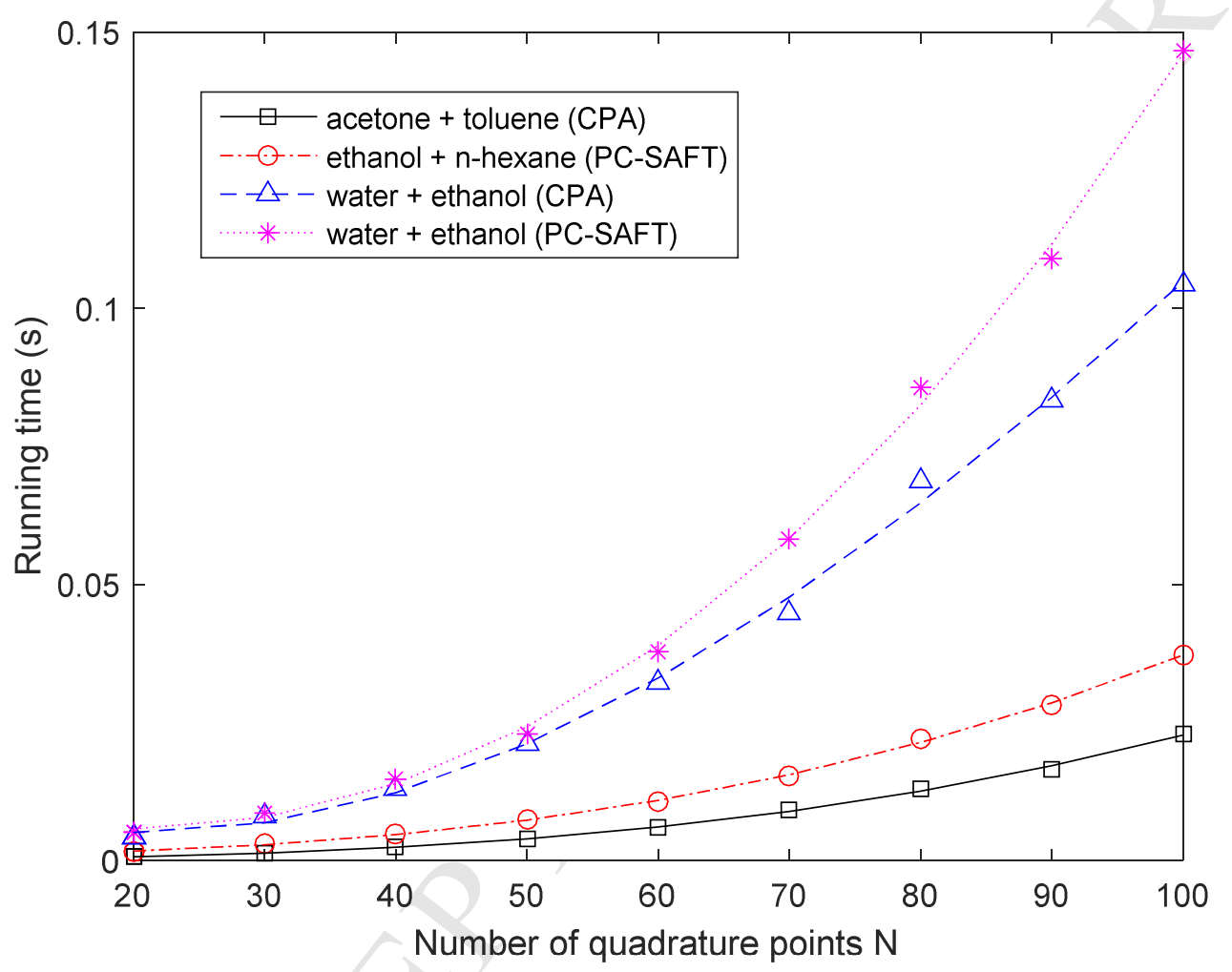

Figure 8 . The relationship between the running time and the number of quadrature points $\mathrm{N}$ for the same systems of Figure 7. The adjustable parameter $\beta_{i j}$ is set to 0.5 for all the cases, and the running time is calculated by Equation (46) for each number of quadrature points with $n r=100$. 
The running time depends on the compiler and computer processor very much. For example, the running time could be $30 \%$ less in a desktop with CPU @3.30 GHz. Therefore, it would be instructive to compare the running time of the proposed algorithm with that used for solving the GM-DGT. In this work, two algorithms are adopted for solving the GM-DGT. One method is employing the phase envelope technique developed by Michelsen [27,28], which automatically determines the number of integration points. It is called the GM-DGT (adapt) approach. The other method is using a fixed number of points with equal interval to discretize the problem domain. It is called the GM-DGT (equal) approach, and it has been commonly used by others $[5,9,25]$. In this work, the implementation of the GM-DGT (adapt) approach is relatively conservative, as at least 22 points are needed by limiting the maximum change of each step, and 500 points are used in the GM-DGT (equal) approach. Some details can be found in the Appendix A and our previous work [19]. The proposed algorithm in this work is denoted as DirectOpt, which represents the Direct Optimization approach. The results are listed in Table 4 for the aforementioned three systems. On average, the running times of each point are less than $0.001 \mathrm{~s}, 0.003 \mathrm{~s}$ and $0.004 \mathrm{~s}$ respectively for systems acetone + toluene, ethanol $+\mathrm{n}$-hexane and water + ethanol, which is consistent with Figure 7. It is somewhat surprising to see that the proposed algorithm is more efficient than the approach GM-DGT (equal). It is also found that the phase envelope technique, the approach GM-DGT (adapt), significantly improves the efficiency of solving the GM-DGT, which is around 6-7 times faster than the approach GM-DGT (equal) in these cases. It needs to point out that a more aggressive procedure without limiting the maximum change of each step will further improve the efficiency. Therefore, the approach GM-DGT (adapt) is recommended to calculate the interfacial tension when the adjustable parameter $\beta_{i j}=0$ is used.

Table 4. Comparison of the running times from different approaches*

\begin{tabular}{ccccc}
\hline Approach & $\begin{array}{c}\text { acetone + toluene } \\
\text { CPA }\end{array}$ & $\begin{array}{c}\text { ethanol + n-hexane } \\
\text { PC-SAFT }\end{array}$ & $\begin{array}{c}\text { water + ethanol } \\
\text { CPA }\end{array}$ & $\begin{array}{c}\text { water + ethanol } \\
\text { PC-SAFT }\end{array}$ \\
\hline DirectOpt & 7 & 23 & 34 & 40 \\
GM-DGT (equal) & 15 & 32 & 40 & 42 \\
GM-DGT (adapt) & 2 & 5 & 6 & 6 \\
\hline
\end{tabular}

The presented running time is the total time of running the cases with composition of the first component from 0.01 to 0.99 with a step 0.01 , and the calculation is repeated 100 times for each point. 
The performance of the proposed algorithm is further investigated for a five-hydrocarbon system, which consists of 0.8205 mole methane, 0.0895 mole propane, 0.05 mole n-pentane, 0.0199 mole $\mathrm{n}$-decane and 0.0201 mole $\mathrm{n}$-hexadecane at $313.15 \mathrm{~K}$. The PR EOS with $\beta_{i j}=0$ is used in this investigation. The calculated interfacial tensions from different approaches are compared with the experimental data in Figure 9. It can be readily seen that the GM-DGT, solving nonlinear algebraic equations, and the proposed algorithm, directly minimizing the interfacial tension, give the same results. This also verifies the proposed algorithm.

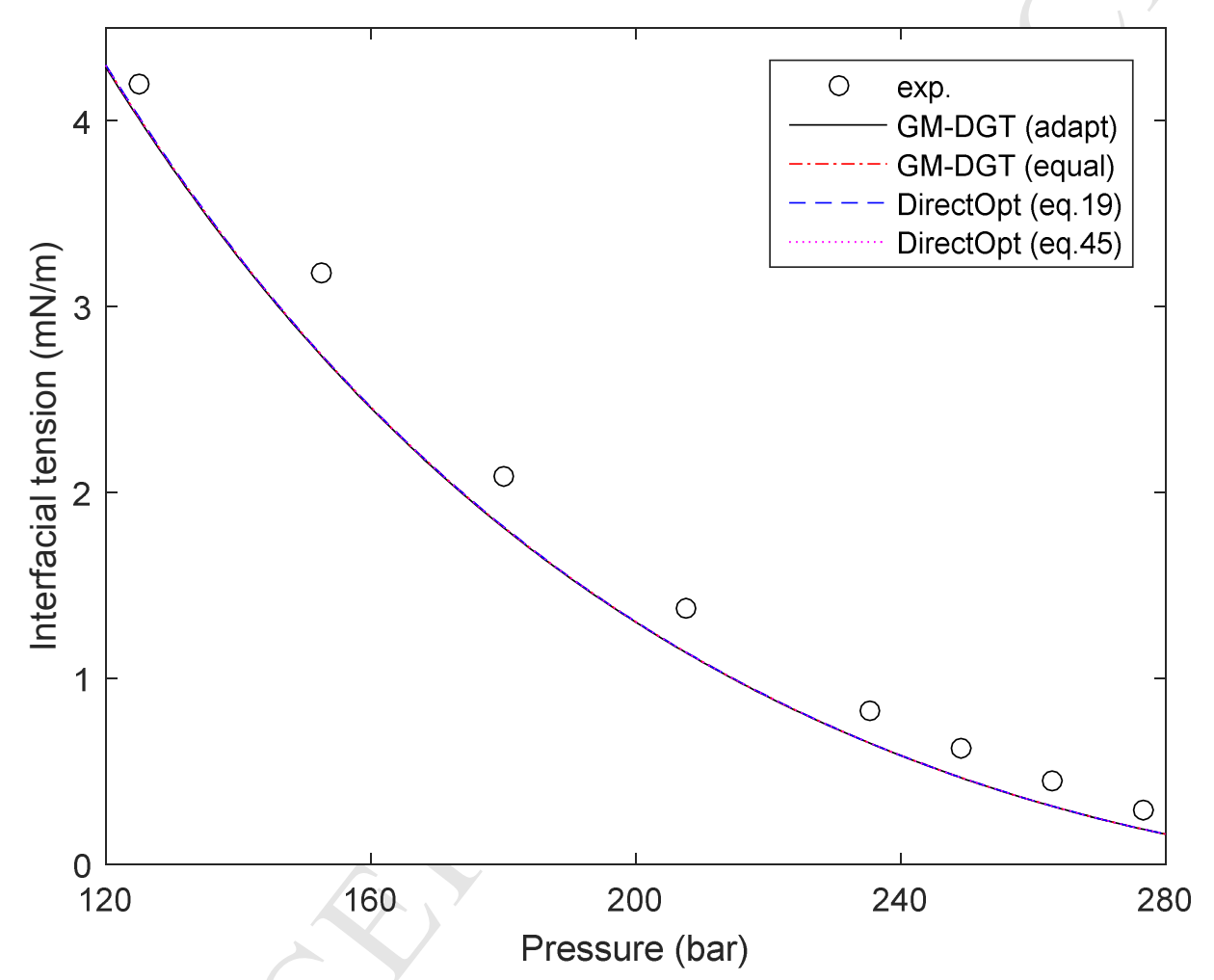

Figure 9. The calculated interfacial tension of a five-hydrocarbon system at $313.15 \mathrm{~K}$ from different approaches with the PR EOS. Experimental data are taken from Danesh et al. [45]. 
The running time of the GM-DGT (adapt) is presented in Figure 10 (Top). In order to eliminate the uncertainty to a large extent, the calculation of every point has been repeated 1000 times. It is somewhat surprising to see that it takes less than 0.001s for the GM-DGT (adapt) to calculate the interfacial tension for every point in the entire investigated range of pressure. It takes slightly longer time as the pressure increases. The comparison of the running times from different approaches is also given in Figure 10 (Bottom). It can be seen that the proposed algorithm and the GM-DGT (equal) show comparable performance, i.e. 5-10 times slower than the GM-DGT (adapt). It needs to point out, however, that the running time is less than $0.007 \mathrm{~s}$ for calculating one point with the proposed algorithm in the entire range of pressure.
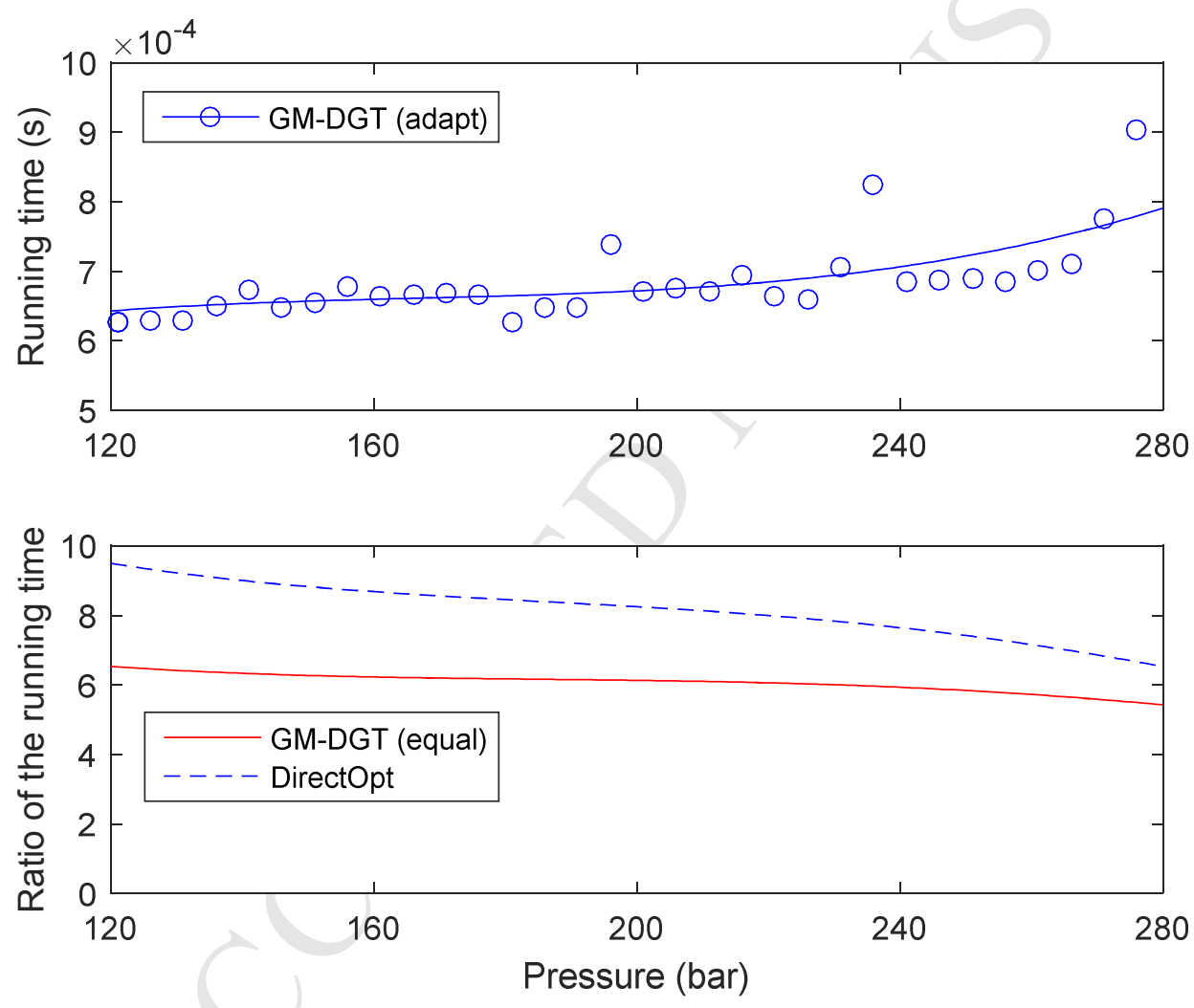

Figure 10. (Top) The running time of the GM-DGT (adapt) for the five-hydrocarbon system at 313.15K with the PR EOS. (Bottom) The ratio of the running times of the GM-DGT (equal) and the DirectOpt with respect to that of the GM-DGT (adapt). The calculation of each point has been repeated 1000 times to obtain the average running time. The machine information of the computer can be found in the text. 


\section{Conclusion}

The DGT considers that the Helmholtz free energy density of an interface consists of an energy term and a gradient term. In this work, we have shown for a planar interface that the gradient term is always larger than the energy term and the difference keeps constant along the interface for a given finite interface width, and the difference of these two terms is becoming smaller as the interface width increases. Based on these findings, we have proposed an inner-outer loop numerical procedure to calculate the interfacial tension of a planar interface within the DGT framework. The outer loop deals with the relationship of the interfacial tension and the interface width. It starts with a small enough interface width, in which linear density profiles provide good estimates, and increases the interface width when the integrals of the energy term and the gradient term are not close enough. In the inner loop, the integrals are evaluated by a combination of the Gauss-Lobatto quadrature and polynomial approximations, and the interfacial tension is minimized by using the restricted step method, in which the densities at the quadrature points are the independent variables. It needs to point out that the direct optimization approach can be replaced by solving the coupled second-order differential equations, and equally good performance might be obtained with proper treatments. The initial estimates for the consecutive iteration steps in the outer loop are generated from the sensitivity of the density profiles with respect to the interface width, and the interface width is adaptively updated based on the easiness of converging the inner loop. In this way, it permits us to obtain accurate results from finite width calculations. Moreover, a much better approximation of the interfacial tension from a path integration along the density profiles is proposed.

There are four key parameters in the algorithm, and the default values are recommended: 20 for the number of the quadrature points, $1.0 \mathrm{~nm}$ for the initial interface width, 0.01 and 0.02 for the convergence criteria respectively for the inner loop and the outer loop. If the algorithm fails with the default parameters, it could be restarted after adjusting one or more parameters. For example, if the algorithm fails at the first iteration step in the outer loop, smaller initial interface width or a better approximation of the initial density profiles could be tried. Otherwise, it is recommended that the number of quadrature points is adjusted before changing the convergence criteria for the inner and outer loops, since they are considered adequate for most practical applications. If the details of the density profiles are crucial, more quadrature points are recommended. 
The efficiency of the algorithm is investigated for three binary systems and one multicomponent system. The efficiency is affected by the thermodynamic model, the complexity and the condition of the system, while the running time is less than $0.007 \mathrm{~s}$ for all the investigated systems. It has also been shown that the proposed algorithm could be considered as efficient as solving the GM-DGT with the equidistant discretization approach, while it may be 5-10 times less efficient than solving the GM-DGT when the phase envelope technique is adopted.

\section{Acknowledgment}

The authors thank Prof. Georgios M. Kontogeorgis for many useful discussions. X. Liang wishes to thank Dr. Xingqing Xiao (NC State University, USA) for many useful comments.

\section{Author Information}

Corresponding Author

* Tel.: 045-45252877. Fax: 045-45882258. Email: xlia@kt.dtu.dk 


\section{List of Symbols}

DGT $=$ Density Gradient Theory

GM-DGT $=$ Geometric-Mean Density Gradient Theory

DirectOpt $=$ Direct Optimization

EOS $=$ Equation $(s)$ Of State

$\mathrm{PR}=$ Peng-Robinson

$\mathrm{CPA}=$ Cubic Plus Association

PC-SAFT $=$ Perturbed Chain Statistical Associating Fluid Theory

$F=$ Helmholtz free energy $(\mathrm{J})$

$\mathrm{T}=$ Temperature $(\mathrm{K})$

$\mathrm{P}=$ Pressure $(\mathrm{Pa})$

$\mathrm{R}$ = Ideal gas constant $(\mathrm{J} / \mathrm{mol}-\mathrm{K})$

$\rho=$ Molar density $\left(\mathrm{mol} / \mathrm{m}^{3}\right)$

$\mu_{i}=$ Chemical potential of component $i(\mathrm{~J} / \mathrm{mol})$

$T_{c}=$ Critical temperature $(\mathrm{K})$

$P_{c}=$ Critical pressure $(\mathrm{Pa})$

$\omega=$ Acentric factor $(-)$

$\Gamma=$ The scaled van der Waals energy parameter in the CPA EOS $(\mathrm{K})[=a / R b]$

$b=$ The co-volume parameter $(\mathrm{L} / \mathrm{mol})$

$\sigma=$ The size of the segment in the PC-SAFT EOS $(\AA)$

$\epsilon / k=$ The energy parameter in the PC-SAFT EOS (K)

$\varepsilon^{H B} / k=$ Association energy $(\mathrm{K})$

$\kappa^{H B}=$ Association volume (-)

$\mathrm{k}_{\mathrm{ij}}=$ Binary interaction parameter for the van der Waals energy parameter (-)

$A=$ Area $\left(\mathrm{m}^{2}\right)$

$\gamma=$ Surface tension $(\mathrm{N} / \mathrm{m})$

$\mathrm{c}_{\mathrm{i}}=$ Influence parameter of molecule $\mathrm{i}\left(\mathrm{J} \cdot \mathrm{m}^{5} / \mathrm{mol}^{2}\right)$

$\mathrm{c}_{\mathrm{ij}}=$ Cross influence parameter between molecules i and $\mathrm{j}\left(\mathrm{J} \cdot \mathrm{m}^{5} / \mathrm{mol}^{2}\right)$

$\beta_{i j}=$ Adjustable parameters used in the combining rule of influence parameters (-)

$t=$ The energy term (tangent plane distance density function) $\left(\mathrm{J} / \mathrm{m}^{3}\right)$ 
$\mathrm{g}=$ The gradient term $\left(\mathrm{J} / \mathrm{m}^{3}\right)$

$\mathrm{G}=$ The scaled gradient term $(\mathrm{J} \cdot \mathrm{m})$

$I_{t}=$ Integral of the energy term $(\mathrm{N} / \mathrm{m})$

$i_{t}=$ Scaled integral of the energy term $\left(\mathrm{N} / \mathrm{m}^{2}\right)$

$I_{g}=$ Integral of the gradient term $(\mathrm{N} / \mathrm{m})$

$i_{g}=$ Scaled integral of the energy term $(\mathrm{N})$

$L=$ Interface width (nm)

$\mathrm{x}=$ Spatial dimension $(\mathrm{nm})$

$\mathrm{Z}=$ Scaled spatial dimension

$L^{\text {init }}=$ Initial interface width for starting the algorithm(nm)

$d L=$ Change of the interface width at each iteration step in the outer loop (nm)

$e=$ Convergence criterion of the inner loop

$\tau=$ Convergence criterion of the outer loop

$w_{k}=$ Quadrature weigths at quadrature point $\mathrm{k}$

$\mathcal{L}_{k}=$ Lagrange polynomials at quadrature point $\mathrm{k}$

$A_{m k}=$ Element of the coefficient matrix of derivatives of the Lagrange polynomials

$\boldsymbol{f}=$ Total gradient of the optimization problem

$\underline{\boldsymbol{H}}=$ Hessian matrix of the optimization problem

$\lambda=$ Correction number for nonpositive definitness matrix

$I=$ Identity matrix or unit matrix

$\delta_{m n}=$ The Kronecker delta function

$\mathrm{N}=$ Number of collocation point (-)

$\mathrm{C}=$ Number of component $(-)$

$v=$ Subscript for vapor phase

$l=$ Subscript for liquid phase

$i, j=$ Subscripts for components

$k, m, n=$ Subscripts for quadrature points 


\section{Appendix A. Solution procedures for the GM-DGT}

If the geometric-mean combining rule is used for the cross influence parameter, the following new variable is introduced,

$$
s=\sum_{j} \sqrt{c_{j}} \rho_{j}
$$

It is called the path function. The basic equation (eq. 10 in the main text) for the density profiles can be rearranged to the following equation (by dividing $\sqrt{c_{i}}$ on both sides),

$$
\frac{\mu_{i}-\mu_{i 0}}{\sqrt{c_{i}}}=\sum_{j} \sqrt{c_{j}} \frac{d^{2} \rho_{j}}{d z^{2}}=\frac{d^{2} s}{d z^{2}}=\alpha
$$

Now we have $\mathrm{NC}+1$ equations (A.1 and A.2) and $\mathrm{NC}+1$ variables $\left(\left\{\rho_{j}\right\}, \alpha\right)$. We also know the boundary values of the path function. If we vary the path function from one phase to the other and solve these equations, we obtain the density profiles. If equidistant points are used for discretizing the path function, it is the approach GM-DGT (equal) in the main text, and we use 500 points in this work. The required information is the same as the traditional approach of choosing a reference component. It is, however, more robust to handle the situation with a nonmonotonic density profile of the reference component.

If we add one more equation,

$$
X=X^{\text {spec }}
$$

where $X$ could be the path function, the variable $\alpha$ or the density of one component. We realize that this set of equations have the same structure for constructing the phase envelope. In this way, it becomes easier for us to vary and control the step and to even solve problems having the nonmonotonic path function. More details of the phase envelope algorithm can be found in the book of Michelsen and Mollerup [28]. This approach is called GM-DGT (adapt) in the main text, and we control the step to have at least 22 points. The Newton-Raphson method is used to solve these equations to an accuracy of $1.0 \mathrm{e}-14$. The other equations, e.g. calculating the interfacial tension after solving the density profiles, can be found in our previous work [19]. 


\section{Appendix B. A further comment on the system of ethanol and $n$-hexane}

As discussed in the previous works [19,20], the system of ethanol and n-hexane is challenging for the DGT with both the CPA and PC-SAFT EOS, and the GM-DGT with the CPA EOS show numerical failures over wide range of composition. The impact of $\beta_{i j}$ on the interfacial tension of this system has been investigated for both models, as presented in Figure B.1. The adjustable parameter $\beta_{i j}$ shows similar impact, and apparently it cannot help to obtain satisfactory correlation of the interfacial tension with the two models by using the given pure component parameters. On one hand, it might be necessary to develop new pure component parameters and/or binary interaction parameters to match this set of experimental data. It would be very helpful, on the other hand, if more experimental data can be measured for the system at the same or different conditions.

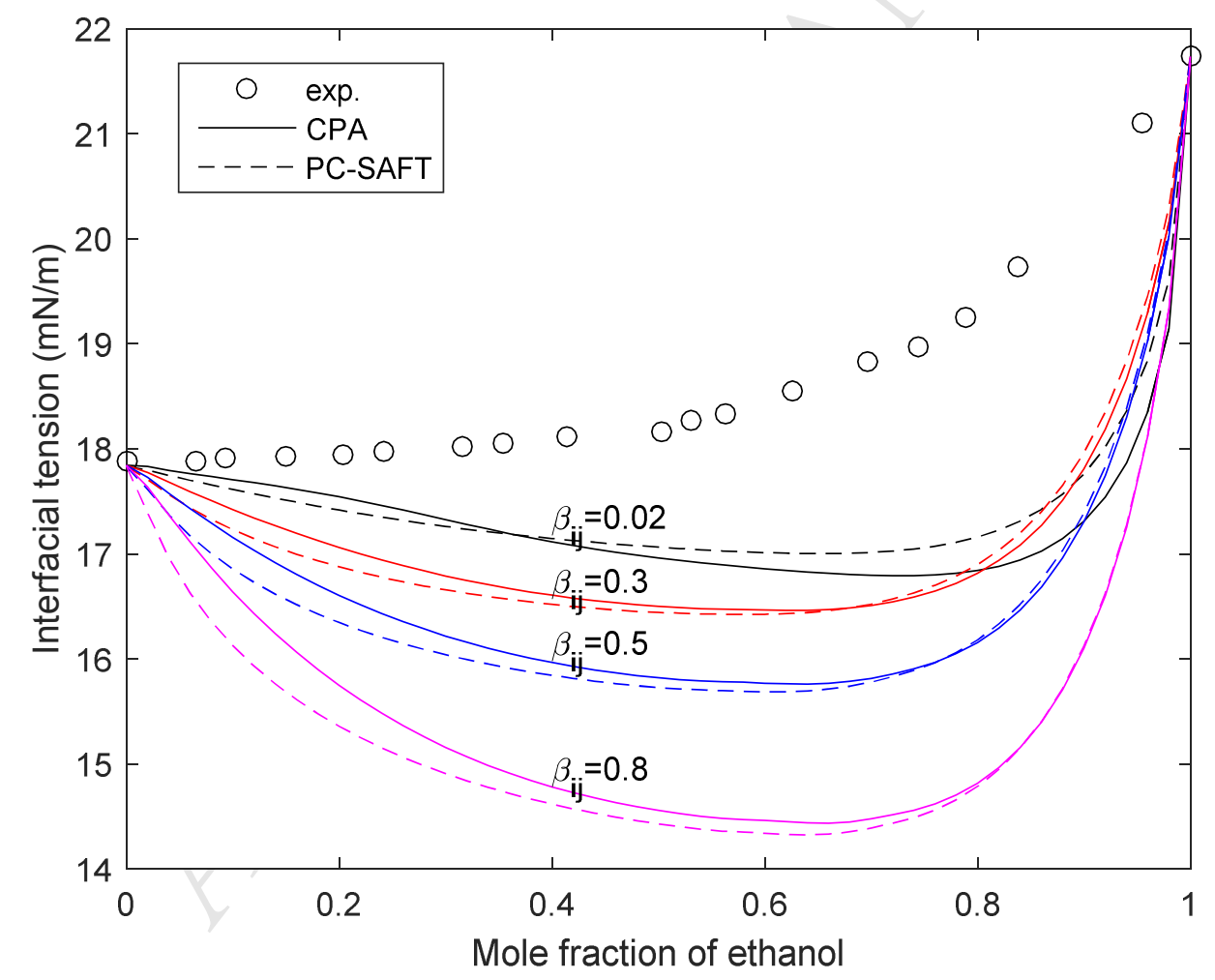

Figure B.1. The interfacial tension of ethanol and n-hexane at $298.15 \mathrm{~K}$ from the CPA and PCSAFT EOS with different adjustable parameters for the cross influence parameters. The experimental data of ethanol + n-hexane are from Jimenez et al. [46]. 
Very recently, Mairhofer et al. [25] have reported the prediction of the interfacial tension of this system from the DFT with the PC-SAFT EOS, which show better results than those from the GM-DGT with the PC-SAFT EOS in the range of low to medium concentration of ethanol. Unfortunately, the DFT overpredicts the interfacial tension of pure ethanol. As shown in Figure B.2, if we readjust the influence parameter of ethanol to $7.0 \times 10^{-20} \mathrm{~J} \cdot \mathrm{m}^{5} / \mathrm{mol}^{2}$, the DGT with the PC-SAFT EOS gives the interfacial tension of pure ethanol close to the one reported by Mairhofer et al. [25], around $26 \mathrm{mN} / \mathrm{m}$. Not surprisingly, the DGT with zero $\beta_{\mathrm{ij}}$ can give quite similar results to those from the DFT [25] in the entire range of composition. Therefore, it is still hard to conclude which framework, the DGT or the DFT, is more suitable for this system with the current available parameters, data and results.

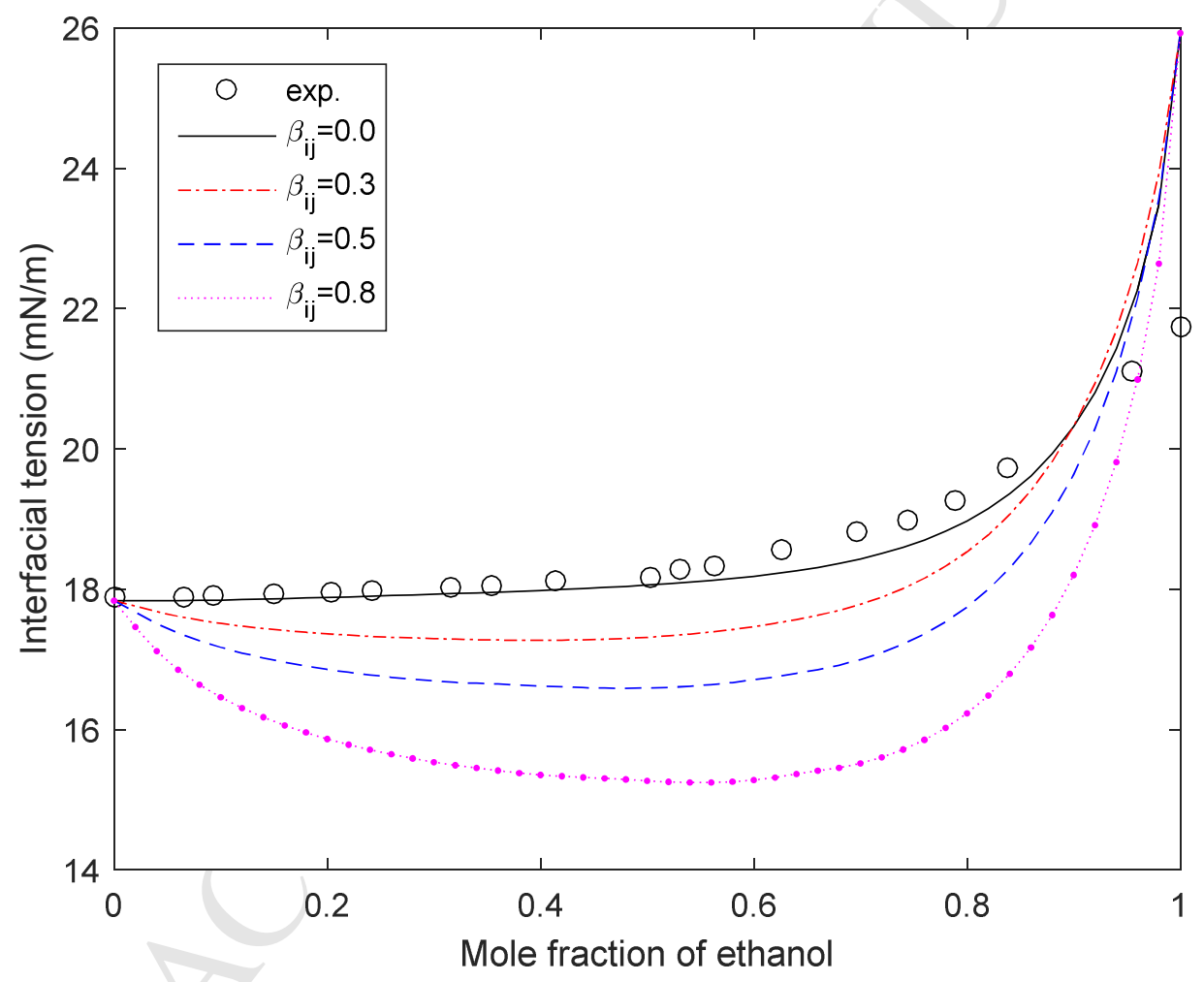

Figure B.2. The interfacial tension of ethanol and n-hexane at $298.15 \mathrm{~K}$ from the PC-SAFT EOS with different adjustable parameter $\beta_{i j}$ values. The influence parameter of ethanol is set to $7.0 \times 10^{-20} \mathrm{~J} \cdot \mathrm{m}^{5} / \mathrm{mol}^{2}$. 


\section{Reference}

[1] B.S. Carey, The gradient theory of fluid interfaces, Ph.D. thesis, University of Minnesota, Minessota, 1979.

[2] M. Sahimi, B.N. Taylor, J. Chem. Phys. 95 (1991) 6749-6761.

[3] Y.X. Zuo, E.H. Stenby, J. Colloid. Int. Sci. 182 (1996) 126-132.

[4] Y.X. Zuo, E.H. Stenby, J. Chem. Eng. (Japan), 29 (1996) 159-165.

[5] P.M.W. Cornelisse, The squared gradient theory applied. Simultaneous modelling of interfacial tension and phase behavior. Ph.D. thesis, TU Delft, The Netherlands, 1997.

[6] H. Kahl, S. Enders, Fluid Phase Equilib. 172 (2000) 27-42.

[7] C. Miqueu, B. Mendiboure, A. Graciaa, J. Lachaise, Fluid Phase Equilib. 207 (2003) 225-246.

[8] C. Miqueu, B. Mendiboure, A. Graciaa, J. Lachaise, Fluid Phase Equilib. 218 (2004) 189-203.

[9] C. Miqueu, B. Mendiboure, A. Graciaa, J. Lachaise, Ind. Chem. Eng. Res. 44 (2005) 3321 3329.

[10] A.J. Queimada, C. Miqueu, I.M. Marrucho, G.M. Kontogeorgis, J.A.P. Coutinho, Fluid Phase Equilib. 228-229 (2005) 479-485.

[11] H. Lin, Y.Y. Duan, Q. Min, Fluid Phase Equilib. 254 (2007) 75-90.

[12] S. Enders, H. Kahl, Fluid Phase Equilib. 263 (2008) 160-167.

[13] O.G. Niño Amézquita, S. Enders, P.T. Jaeger, R. Eggers, J. Supercrit. Fluids. 55 (2010) 724-734.

[14] O.G. Niño Amézquita, S. Enders, P.T. Jaeger, R. Eggers, Ind. Eng. Chem. Res. 49 (2010) 529-601.

[15] V. Vinš, B. planková, J. Hrubý, Int. J. Thermophys. 34 (2013) 798-812.

[16] S. Khosharay, M. Abolala, F. Varaminian, J. Mol. Liq. 198 (2014) 292-298.

[17] Y.T.F. Chow, D.K. Eriksen, A. Galindo, A.J. Haslam, G. Jackson, G.C. Maitlan, J.P.M. Trusler, Fluid Phase Equilib. 2015. 
[18] P.M. Larsen, B.M. Mogensen, G.M. Kontogeorgis, Fluid Phase Equilib. 408 (2016) 170-179.

[19] X.D. Liang, M.L. Michelsen, G.M. Kontogeorgis, Fluid Phase Equilib. 415 (2016) 75-83.

[20] X.D. Liang, M.L. Michelsen, G.M. Kontogeorgis, Fluid Phase Equilib. 428 (2016) 153-163.

[21] G.L. Shen, C. Held, X.H. Lu, X.Y. Ji, Mol. Phys. 114 (2016) 2492-2499.

[22] L.M.C. Pereira, A. Chapoy, R. Burgass, M.B. Oliveira, J.A.P. Coutinho, B. Tohidi, J. Chem. Thermodyn. 93 (2016) 404-415.

[23] J.S. Rowlinson, Translation of J.D. van der Waals" "The Thermodynamic theory of capillarity under the hypothesis of a continuous variation of density". J. Stat. Phys. 20 (1979) 197-200.

[24] J.W. Cahn, J.E. Hilliard, J. Chem. Phys. 28 (1958) 258-267.

[25] J. Mairhofer, J. Gross, Fluid Phase Equilib. 439 (2017) 31-42.

[26] J.S. Kou, S.Y. Sun, X.H. Wang, Comput. Methods Appl. Mech. Engrg. 292 (2015) 92-106.

[27] M.L. Michelsen, Fluid Phase Equilib. 23 (1985) 181-192.

[28] M.L. Michelsen, J.M. Mollerup, Thermodynamic Models: Fundamentals \& Computational Aspects; Tie-Line Technology: Holte, Denmark, 2007.

[29] Z.H. Qiao, S.Y. Sun, SIAM J. Sci. Comput. 36 (2014) 708-728.

[30] X.Q. Mu, F. Frank, F.O. Alpak, W.G. Chapman, Fluid Phase Equilib. 435 (2017) 118-130.

[31] J.Villadsen, M.L. Michelsen, Solution of differential equation models by polynomial approximation. Prentice-Hall, Inc.: New Jersey, 1978.

[32] R. Fletcher, Practical methods of optimization. John Wiley and Sons: New York, 2000.

[33] D.Y. Peng, D.B. Robinson, Ind. Eng. Chem. Fundam. 15 (1976) 59-65.

[34] G.M. Kontogeorgis, E.C. Voutsas, I.V. Yakoumis, D.P. Tassios, Ind. Chem. Eng. Res. 35 (1996) 4310.

[35] N. von Solms, M.L. Michelsen, G.M. Kontogeorgis, Ind. Eng. Chem. Res. 42 (2003) 10981105 . 
[36] G.M. Kontogeorgis, G.K. Folas, Thermodynamic models for industrial applications - from classical and advanced mixing rules to association theories. John Wiley and Sons: New York, 2010.

[37] G.M. Kontogeorgis, I.V. Yakoumis, H. Meijer, E. Hendriks, T. Moorwood, Fluid Phase Equilib. 158-160 (1999) 201-209.

[38] G.K. Folas, J. Gabrielsen, M.L. Michelsen, E.H. Stenby, G.M. Kontogeorgis, Ind. Chem. Eng. Res. 44 (2005) 3823-3833.

[39] G.K. Folas, G.M. Kontogeorgis, M.L. Michelsen, E.H. Stenby, Ind. Chem. Eng. Res. 45 (2006) 1516-1526.

[40] I.V. Yakoumis, G.M. Kontogeorgis, E.C. Voutsas, D.P. Tassios, Fluid Phase Equilib. 130 (1997) 31-47.

[41] X.D. Liang, I. Tsivintzelis, G.M. Kontogeorgis, Ind. Chem. Eng. Res. 53 (2014) 1449314507.

[42] X.D. Liang, K. Thomsen, W. Yan, G.M. Kontogeorgis, Fluid Phase Equilib. 360 (2013) 222-232.

[43] J. Gross, G. Sadowski, Ind. Eng. Chem. Res. 40 (2001) 1244-1260.

[44] Ø. Wilhelmsen, D. Bedeaux, D. Reguera, J. Chem. Phys. 142 (2015) 064706.

[45] A.S. Danesh, A.Y. Dandekar, A.C. Todd, R. Sarkar, SPE Annual Technical Conference and Exhibition, Dallas, Texas, 6-9 October, 1991. SPE 22710.

[46] E. Jimenez, H. Casas, L. Segade, C. Franjo, J. Chem. Eng. Data, 45 (2000) 862-866. 